\title{
Personalized individual semantics-based approach for linguistic failure modes and effects analysis with incomplete preference information
}

\begin{abstract}
Failure modes and effects analysis (FMEA) is a very useful reliability-management instrument for detecting and mitigating risks in various fields. Linguistic assessment approach has recently been widely used in FMEA. Words mean different things to different people, so FMEA members may present personalized individual semantics (PIS) in their linguistic assessment information. This paper designs a PIS-based FMEA approach with members expressing their opinions over failure modes and risk factors using linguistic distribution assessment matrices (LDAMs) and also provide their opinions over failure modes using incomplete additive preference relations (APRs). A preference information preprocessing method with a two-stage optimization model is presented to generate complete APRs with acceptable consistency levels from incomplete APRs. Then, a deviation minimum-based optimization model is designed to personalize individual semantics by minimizing the deviation between APR and the numerical assessment matrix derived from the corresponding LDAM. This is followed by the developing of a ranking process to generate the risk ordering of failure modes. A case study and a detailed comparison analysis are presented to show the effectiveness of the PIS-based linguistic FMEA approach.
\end{abstract}

Keywords: Reliability management; failure modes and effects analysis; personalized individual semantics; consistency; optimization

\section{Introduction}

Failure modes and effects analysis (FMEA) is a very powerful reliability-management instrument, which is frequently used in product design to identify the most critical causes of a product's failure and to mitigate their risks $[3,9,12,15,29,32,36]$. Thus, risk assessment and prioritization of failure modes are key issues in FMEA [4, 5, 12, 40]. Traditionally, the risk assessment information on each failure mode with respect to the three risk factors of occurrence $(\mathrm{O})$, severity $(\mathrm{S})$, and detection $(\mathrm{D})$ is measured using a (1-10) numerical points scale. The product of the $(\mathrm{O}, \mathrm{S}, \mathrm{D})$ numerical risks values is defined as the risk priority number (RPN) of a failure mode [39], which is subsequently used to produce a risk ordering of failure modes. Different levels of security control measures for failure modes are implemented in order to mitigate risk, and failure modes with high RPN values are paid more attention. Thus, the ultimate decision result in an FMEA method/model is the risk ordering of failure modes by their RPN values. For a comprehensive introduction of the FMEA implementation process, please refer to Refs. [22, 39]. 
The RPN-based FMEA approach has been associated with several issues (see [1, 2, 10, 11, 23, $37,38,41,46])$, being particularly relevant to the present paper that FMEA members are obliged to express accurate risk assessment information using the aforementioned 1-10 numerical points scale. Indeed, in some real-world decision processes, FMEA members may prefer or feel more comfortable assessing risk using linguistic rather than numerical values (e.g., [16, 21, 40]). To address this issue, the following different linguistic FMEA approaches have been developed/proposed to date: (i) interval two-tuple linguistic risk assessments and the ELECTRE (Elimination Et Choix Traduisant la REalite) approach for ranking failure modes [24]; (ii) triangular fuzzy linguistic risk assessments, the Choquet integral and prospect theory [41]; (iii) the linguistic weighted geometric operator and fuzzy priority methodology [47]; (iv) linguistic distribution of risk assessment information with an improved TODIM (Portuguese acronym for 'interactive and multi-criteria decision making') approach to yield the risk ordering of failure modes [14]; (v) multi-granular linguistic distribution risk assessments to model uncertain opinions of FMEA members [34]; (vi) consensus-based group decision-making (GDM) approaches for FMEA with linguistic distribution risk assessments [44, 45].

These linguistic FMEA approaches represent a great progress because they provide a more flexible framework than the previous numerical approaches. However, they still do not accommodate all possible real scenarios because they are based on the premise that the linguistic labels (words) implemented/used mean the same for all FMEA members, when the reality is that, in general, words may mean different things to different individuals, a phenomenon referred to as personalized individual semantics (PIS) that affects practical FMEA problems decision results ([17-19, 30, 31]). Indeed, when assessing the risk level of a failure mode, two FMEA members may assess the risk level of the failure mode with the same word "high" but with distinct semantics. As it is illustrated later in this paper in Section 3.1 (Example 1), ignoring the PIS issue affects the reliability management quality.

To the best of our knowledge, PIS has not been considered yet in the existent linguistic FMEA approaches, which is the goal of this paper. Thus, this paper aims at developing the mathematical framework for managing PIS in the linguistic FMEA problem to improve the reliability management quality. This is achieved by means of the following two main research objectives:

(1) To implement a general linguistic decision context to formulate the PIS-based FMEA problem, which is based on the mathematical representation of the FMEA members' preference information using (i) the general linguistic model of linguistic distribution assessment matrices (LDAMs) [6, 14, 20, 28, 42, 43] for the risk assessment information on the failure modes with respect to the risk factors $(\mathrm{O}, \mathrm{S}, \mathrm{D})$; and (ii) general incomplete additive preference relations 
(APRs) on the failure modes.

(2) To obtain the resolution procedure to deal with the PIS-based linguistic FMEA problem: (i) First, a preference information preprocessing method based on a two-stage optimization model is constructed to generate complete APRs with acceptable consistency levels from the incomplete APRs; (ii) Second, inspired by the numerical scale function-based approaches for dealing with PIS reported in [17-19], a deviation minimum-based optimization model is proposed to personalize individual semantics in the LADMs, which seeks to minimize the deviation between the APR and the numerical assessment matrix (NAM) derived from the LDAM using a PIS-based numerical scale (PNS); (iii) Third, a ranking process is presented to generate the risk ordering of failure modes from the obtained NAMs and APRs.

Finally, a validation study is reported with a detailed comparison analysis between the proposed PIS-based linguistic FMEA approach and the fixed numerical scale (FNS) based linguistic FMEA approach, which is complemented with the exemplification of its practical use with a case study related to the problem of the reliability management of blood transfusion.

The remainder of this paper is arranged as follows. Section 2 includes the necessary preliminary concepts to make this paper self-contained. Section 3 presents a motivation example and formulates the PIS-based linguistic FMEA problem. Section 4 designs the detailed solution procedure for the PIS-based linguistic FMEA problem. Following this, Section 5 illustrates the practical use of the PIS-based linguistic FMEA approach with the above mentioned case study. Subsequently, Section 6 presents a comparison analysis to show the effectiveness of the PIS-based linguistic FMEA approach. Finally, Section 7 concludes the paper and discusses future research directions.

In order to improve readability, all acronyms and notations used within the paper are included in Appendix A.

\section{Preliminaries}

This section introduces basic knowledge regarding APRs, the linguistic distribution assessments, and the numerical scale function of a linguistic term set.

\section{(1) Additive preference relations (APRs)}

Let $X=\left\{x_{1}, x_{2}, \ldots, x_{n}\right\}$ be a set of objects. The concept of APR over $X$ and its consistency level are provided in the below definitions:

Definition 1 [13]. An APR on a set of objects $X$ is represented by a matrix, $A=\left(a_{i j}\right)_{n \times n}$, in which its element $a_{i j} \in[0,1]$ represents the preference intensity of object $x_{i}$ over object $x_{j}$, subject to the following reciprocity property: $a_{i j}+a_{j i}=1 \quad \forall i, j \in\{1,2, \ldots, n\}$.

Definition 2 [13]. The consistency level of an APR $A=\left(a_{i j}\right)_{n \times n}$ is measured with the 
following $[0,1]$-valued function:

$$
C L(A)=1-\frac{4}{n(n-1)(n-2)} \sum_{i, j, z=1 ; i<j<z}^{n}\left|a_{i j}+a_{j z}-a_{i z}-0.5\right| .
$$

The larger the value of $C L(A)$, the more consistent the APR $A=\left(a_{i j}\right)_{n \times n}$ is. In particular, $A=\left(a_{i j}\right)_{n \times n}$ is completely consistent when $C L(A)=1$. Let $\alpha \in[0,1]$ be a predefined threshold value used to judge whether the consistency of $A=\left(a_{i j}\right)_{n \times n}$ is acceptable or not, i.e. when $C L(A) \geq \alpha$, then APR $A=\left(a_{i j}\right)_{n \times n}$ is of acceptable consistency; otherwise, it is not of acceptable consistency. The determination of the parameter $\alpha$ usually depends on the actual situation/problem being dealt with.

The complexity of a particular decision context may lead to a decision maker not been able to provide all elements of an APR $A=\left(a_{i j}\right)_{n \times n}$. In this case, the APR is referred to as an incomplete APR. We use $\dot{A}=\left(\dot{a}_{i j}\right)_{n \times n}$ to denote an incomplete APR. In particular, $\dot{a}_{i j}=$ null if the preference intensity of object $x_{i}$ over object $x_{j}$ is not provided by the decision maker.

\section{(2) Linguistic distribution assessments}

Let $L=\left\{l_{0}, \ldots, l_{g}\right\}$ be a linguistic term set with granularity of $g+1$ and term $l_{j}$ a possible linguistic value subject to the following two conditions: (1) $L$ is ordered: $l_{i} \leq l_{j} \Leftrightarrow i \leq j$, and (2) there is an inverse function such that $n e g\left(l_{j}\right)=l_{g-j}$. The linguistic distribution assessment approach was proposed to deal with uncertain and vague assessment information effectively [43].

The linguistic distribution assessment is formally presented below.

Definition 3 [43]. A distribution assessment of a linguistic term set $L$ is represented as $L A D=\left\{\left(l_{t}, \vartheta_{t}\right) \mid t=0,1, \ldots, g\right\}$, with symbolic proportions $\vartheta_{t} \in[0,1]$ of linguistic terms $l_{t}$ satisfying $\sum_{t=0}^{g} \vartheta_{t}=1$.

\section{(3) Numerical scale function}

The concept of numerical scale function was proposed to transform linguistic terms into real numbers [7], with the aim to facilitate the computational process in the linguistic assessment approach based GDM.

Definition 4. Let $L=\left\{l_{0}, \ldots, l_{g}\right\}$ be a linguistic term set and $R N$ be the set of real numbers. A function $N S: L \rightarrow R N$ is called a numerical scale of $L$, and $N S\left(l_{i}\right)$ is the numerical index of $l_{i}$. If the function $N S$ is strictly monotone increasing, then $N S$ is called an ordered numerical scale of $L$.

In essence, the numerical scale provides numerical meaning of linguistic terms in GDM. When all individuals in a GDM problem have the same numerical indexes of linguistic terms, then the GDM problem is referred to as an FNS-based linguistic GDM. In many cases though, words mean different things to different individuals, i.e. individuals have different PIS, PNSs are employed in 
linguistic GDM, and the GDM problem is referred to as a PIS-based linguistic GDM [17-19].

\section{The PIS-based linguistic FMEA problem: Motivation and problem formulation}

This section includes a motivation example to analyze the influence of PIS on linguistic FMEA. Then, the PIS-based linguistic FMEA problem formulation is put forward.

\subsection{Motivation example: The influence of PIS on linguistic FMEA}

It was argued in the introduction section that the PIS issue may appear in the linguistic assessments of the FMEA members and that ignoring it weakens the reliability management quality. Here, we provide an example that provides evidence to support this argument.

Example 1: Let $L=\left\{l_{0}=\right.$ very low,$l_{1}=$ low,$l_{2}=$ moderately low,$l_{3}=$ moderate , $l_{4}=$ moderate high,$l_{5}=$ high,$l_{6}=$ very high $\}$ be the linguistic term set used by FMEA members $T M_{1}$ and $T M_{2}$ to evaluate the risk level of failure modes $F M_{1}$ and $F M_{2}$. The aim is to find a risk ordering of $F M_{1}$ and $F M_{2}$ based on the linguistic risk assessments provided by $T M_{1}$ and $T M_{2}$, who considered equally important. Let us assume that the risk assessments on $\left(F M_{1}, F M_{2}\right)$ provided by $T M_{1}$ and $T M_{2}$ are $\left(l_{5}\right.$ and $\left.l_{4}\right)$ and $\left(l_{3}\right.$ and $\left.l_{5}\right)$, respectively.

The process to obtain the risk ordering of $F M_{1}$ and $F M_{2}$ is as follows: (1) linguistic assessments are transformed into numerical assessments using a numerical scale function as per Definition 4; (2) the total evaluation value (TEV) of each failure mode is obtained as the weighted average of the numerical assessments of the two FMEA members, which in this case will be the mean value as both members are equally important; (3) the TEV values are be used to generate the risk ordering of $F M_{1}$ and $F M_{2}$.

In the following, two cases are considered:

Case A: PIS is not considered and $T M_{1}$ and $T M_{2}$ use the same following numerical scale function: $\quad N S^{1}\left(l_{i}\right)=N S^{2}\left(l_{i}\right)=i / 6 \quad(i=0,1, \ldots, 6)$. Thus, it is $\quad T E V_{1}=0.5\left(N S^{1}\left(l_{5}\right)+N S^{2}\left(l_{3}\right)\right)=$ $0.5(5 / 6+0.5)=0.6667$ and $T E V_{2}=0.5\left(N S^{1}\left(l_{4}\right)+N S^{2}\left(l_{5}\right)\right)=0.5(2 / 3+5 / 6)=0.75$.

Case B: PIS is considered and $T M_{1}$ and $T M_{2}$ use different numerical scale functions: $\left\{N S^{1, *}\left(l_{0}\right), \ldots, N S^{1, *}\left(l_{6}\right)\right\}=\{0,1 / 6,1 / 3,1 / 2,0.52,0.92,1\}$ and $\left\{N S^{2, *}\left(l_{0}\right), \ldots, N S^{2, *^{*}}\left(l_{6}\right)\right\}=\{0 / 6,1 / 6,1 / 3,1 / 2$, $2 / 3,5 / 6,6 / 6\}$, respectively. Thus, it is $T E V_{1}^{*}=0.5\left(N S^{1, *}\left(l_{5}\right)+N S^{2, *}\left(l_{3}\right)\right)=0.5(0.92+0.5)=0.71$ and $T E V_{2}^{*}=0.5\left(N S^{1, *}\left(l_{4}\right)+N S^{2, *}\left(l_{5}\right)\right)=0.5(0.52+5 / 6)=0.6767$.

In case A, the risk level of $T M_{2}$ is higher than $T M_{1}$. However, the risk level of $T M_{1}$ is higher than $T M_{2}$ in case $\mathrm{B}$. This example shows that ignoring the PIS issue may result in obtaining the opposite risk ordering of failure modes. So, it is worth to design an approach to address the PIS issue in the linguistic FMEA problem when it is present.

\subsection{Problem formulation}

In the following, we formulate the PIS-based FMEA problem in a general linguistic decision 
context, with linguistic distribution assessments and incomplete preference information used to model the uncertain opinions of FMEA members. Formally, the PIS-based linguistic FMEA problem is described as follows.

Let $F M=\left\{F M_{1}, F M_{2}, \ldots, F M_{n}\right\}$ be the set of $n$ potential failure modes, and $R F=\left\{R F_{1}, R F_{2}, \ldots, R F_{y}\right\}$ be the set of $y$ risk factors. Let $\omega=\left(\omega_{1}, \omega_{2}, \ldots, \omega_{y}\right)^{T}$ be the weight vector of risk factors set $R F$, where $\omega_{i} \geq 0 \quad(i=1,2, \ldots, y)$ is the normalized weight of risk factor $R F_{i}$, i.e. $\sum_{i=1}^{y} \omega_{i}=1$. A group of team members $T M=\left\{T M_{1}, T M_{2}, \ldots, T M_{m}\right\}$ from different departments is formed to carry out the failure and risk analysis. Let $\lambda=\left(\lambda_{1}, \lambda_{2}, \ldots, \lambda_{m}\right)^{T}$ be the weight vector of team members set $T M$, where $\lambda_{k} \in[0,1]$ is the normalized weight of team member $T M_{k}$, i.e. $\sum_{k=1}^{m} \lambda_{k}=1$.

In the PIS-based linguistic FMEA problem, each FMEA member $T M_{k} \in T M$ provides two kinds of preference information:

(1) The linguistic assessment of each failure mode $F M_{i}$ with respect each of the risk factors $R F_{j}: u_{i j}^{k}=\left\{\left(l_{s}, g_{i j, s}^{k}\right) \mid s=0,1, \ldots, g\right\}$. Thus, each FMEA member $T M_{k} \in T M$ provides an LDAM $U^{k}=\left(u_{i j}^{k}\right)_{n \times y}$.

(2) The preference intensity of failure mode $F M_{i}$ over failure mode $F M_{j}$, which could be unknown and, as a result, an incomplete APR $\dot{A}^{k}=\left(\dot{a}_{i j}^{k}\right)_{n \times n}$ on the set of failure modes $F M$ is provided by the FMEA member $T M_{k} \in T M$.

Then, there are two key decision problems:

(i) How to personalize individual semantics in the FMEA members' LADMs through PNSs, which will provide a basis for the linguistic FMEA;

(ii) How to find the risk ordering of the failure modes based on the provided LDAMs $\left\{U^{1}, \ldots, U^{m}\right\}$, incomplete APRs $\left\{\dot{A}^{1}, \ldots, \dot{A}^{m}\right\}$ and the PNSs.

\section{Resolution procedure for the PIS-based linguistic FMEA problem}

In this section, we present the detailed resolution procedure for the PIS-based linguistic FMEA problem.

In the PIS-based linguistic FMEA problem, the failure modes assessments are modelled using two kinds of preference information (i.e., LDAMs $\left\{U^{1}, \ldots, U^{m}\right\}$ and incomplete APRs $\left\{\dot{A}^{1}, \ldots, \dot{A}^{m}\right\}$ ), which depict more accurately and comprehensively the evaluation information of FMEA members. Taking LDAMs $\left\{U^{1}, \ldots, U^{m}\right\}$ and incomplete APRs $\left\{\dot{A}^{1}, \ldots, \dot{A}^{m}\right\}$ as input data, the resolution framework of the PIS-based linguistic FMEA is described as a three-step procedure (See Fig. 1): Preference information preprocessing (Step 1); personalizing individual semantics (Step 2); and failure mode ranking (Step 3). 


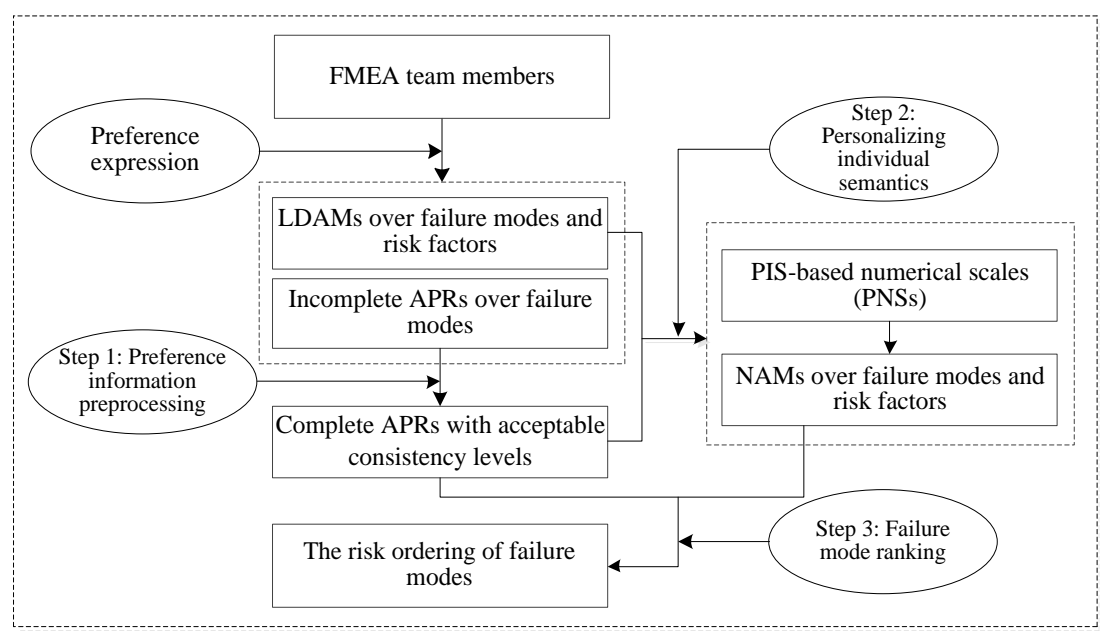

Fig. 1. The resolution framework of the PIS-based linguistic FMEA approach

\section{Step 1: Preference information preprocessing}

In Step 1, a preference information preprocessing is applied to the incomplete APRs $\left\{\dot{A}^{1}, \ldots, \dot{A}^{m}\right\}$ to ensure that the used preference data is reliable. Specifically, a two-stage optimization model to estimate missing values in the incomplete APRs $\left\{\dot{A}^{1}, \ldots, \dot{A}^{m}\right\}$ and to improve their consistency level is proposed.

\section{Step 2: Personalizing individual semantics}

In Step 2, a deviation minimum-based optimization model to personalize individual semantics of FMEA members is constructed to derive the PNSs of linguistic terms and subsequently convert the LDAMs into NAMs.

\section{Step 3: Failure mode ranking}

In Step 3, the risk ordering of failure modes is obtained from the NAMs and complete APRs.

Sections 4.1-4.3 detail fully these three steps, respectively.

\subsection{Preference information preprocessing}

In the preference information preprocessing, missing values in the incomplete APRs are first estimated (Stage 1) followed by the improvement of the consistency level of the completed APRs (Stage 2). The preference information preprocessing ensures that the preference data in APRs is reliable.

Stage 1: The consistency maximum-based optimization model to estimate missing values

Let $\dot{A}^{k}=\left(\dot{a}_{i j}^{k}\right)_{n \times n}$ be an incomplete APR and all its known elements but those in the main diagonal be denoted by $K A^{k}=\left\{\dot{a}_{i j}^{k} \mid \dot{a}_{i j}^{k} \neq\right.$ null for $i, j=1,2, \ldots, n$ and $\left.i \neq j\right\}$. Let $K P^{k}$ be the set of positions of elements in $K A^{k}$, and $U K P^{k}$ be the set of positions of the unknown element, i.e. $K P^{k}=\left\{(i, j) \mid \dot{a}_{i j}^{k} \in K A^{k}\right\}$ and $U K P^{k}=\left\{(i, j) \mid \dot{a}_{i j}^{k}=\right.$ null for $\left.i, j=1,2, \ldots, n\right\}$. In the following, we present a maximization of consistency criterion based methodology [8] to yield a complete APR $A^{k}=\left(a_{i j}^{k}\right)_{n \times n}$ from $\dot{A}^{k}=\left(\dot{a}_{i j}^{k}\right)_{n \times n}$, which will be referred to as the complete APR associated with an 
incomplete APR.

The consistency level as per Definition 2 [13] is used as the objective function of the following consistency maximum-based optimization model to obtain the missing elements of incomplete APRs:

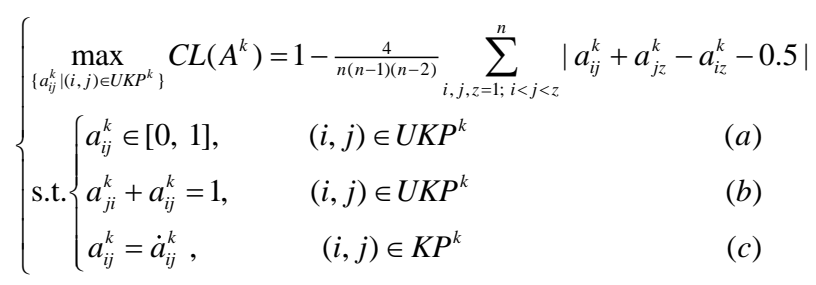

For the sake of convenience, model (2) is denoted as $P_{1}$. In model $P_{1}$, the objective function is to maximize the consistency level of $A^{k}$, and $\left\{a_{i j}^{k} \mid(i, j) \in U K P^{k}\right\}$ are the decision variables. Moreover, as per Definition 1, constraints (a) and (b) guarantee that the decision variables are in the domain $[0,1]$ and that $A^{k}$ satisfies the reciprocity property, respectively, while constraint (c) ensures that the known preference values in $\dot{A}^{k}$ remain unchanged in the process of obtaining the complete APR $A^{k}$.

Model $P_{1}$ is a non-linear programming model. The following proposition provides the equivalent linear programming model to model $P_{1}$.

Proposition 1: By introducing a collection of new variables $b_{i j z}^{k} \in[0,1.5] \quad(i<j<z$; $i, j, z=1,2, \ldots, n)$, the non-linear programming model $P_{1}$ is equivalently converted into the following linear programming model (denoted as $P_{2}$ ):

$$
\left\{\begin{array}{l}
\max _{\left\{a_{i j}^{k} \mid(i, j) \in U K P^{k}\right\}}\left(1-\frac{4}{n(n-1)(n-2)} \sum_{i, j, z=1 ; i<j<z}^{n} b_{i j z}^{k}\right) \\
\text { s.t. }\left\{\begin{array}{l}
a_{i j}^{k}+a_{j z}^{k}-a_{i z}^{k}-0.5 \leq b_{i j z}^{k}, i<j<z ; i, j, z=1,2, \ldots, n \quad(a) \\
-a_{i j}^{k}-a_{j z}^{k}+a_{i z}^{k}+0.5 \leq b_{i j z}^{k}, i<j<z ; i, j, z=1,2, \ldots, n(b) \\
a_{i j}^{k} \in[0,1], \quad(i, j) \in U K P^{k} \\
a_{j i}^{k}+a_{i j}^{k}=1, \quad(i, j) \in U K P^{k} \\
a_{i j}^{k}=\dot{a}_{i j}^{k}, \quad(i, j) \in K P^{k} \\
b_{i j z}^{k} \in[0,1.5], i<j<z ; i, j, z=1,2, \ldots, n
\end{array}\right.
\end{array}\right.
$$

The proof of Proposition 1 is provided in Appendix B.

Proposition 1 provides a linear programming-based approach to obtain the optimal complete $\operatorname{APR} A^{k, *}=\left(a_{i j}^{k, *}\right)_{n \times n}$, i.e. with maximum consistency level, associated with an incomplete APR $\dot{A}^{k}=\left(\dot{a}_{i j}^{k}\right)_{n \times n}$. Generally, linear programming models are very easy to be solved with some software packages (e.g., LINGO). 
Stage 2: The adjustment minimum-based optimization model for consistency improvement

In some situations, the consistency level of the complete APR derived from Stage 1 $A^{k, *}=\left(a_{i j}^{k, *}\right)_{n \times n}$ is not acceptable, i.e., $C L\left(A^{k,{ }^{*}}\right)<\alpha$. To deal with this issue, in Stage 2 an adjustment minimum-based optimization model is applied to generate a complete APR $A^{k}=\left(a_{i j}^{k}\right)_{n \times n}$ with an acceptable consistency level associated with $A^{k, *}=\left(a_{i j}^{k, *}\right)_{n \times n}$. To achieve this, a distance function between two complete APRs $A^{k}=\left(a_{i j}^{k}\right)_{n \times n}$ and $\bar{A}^{k}=\left(\bar{a}_{i j}^{k}\right)_{n \times n}$ regarding the set $K P^{k}$ is required to be defined, which in this paper is computed as below:

$$
k d\left(A^{k}, \bar{A}^{k}\right)=\frac{1}{\left\|K P^{k}\right\|} \sum_{(i, j) \in K P^{k}}\left|a_{i j}^{k}-\bar{a}_{i j}^{k}\right|
$$

where $\left\|K P^{k}\right\|$ denotes the number of elements in $K P^{k}$. It is clear that $k d\left(A^{k}, \bar{A}^{k}\right) \in[0,1]$.

When adjusting APR $A^{k, *}$, we aim for the distance between $A^{k,{ }^{*}}=\left(a_{i j}^{k, *}\right)_{n \times n}$ and $A^{k}=\left(a_{i j}^{k}\right)_{n \times n}$ regarding the set $K P^{k}$ to be as small as possible, that is

$$
\min _{A^{k}} k d\left(A^{k}, A^{k, *}\right)=\frac{1}{\left\|K P^{k}\right\|_{(i, j) \in K P^{k}}} \sum_{i j}^{k}-a_{i j}^{k, *} \mid .
$$

Meanwhile, the consistency level of $A^{k}=\left(a_{i j}^{k}\right)_{n \times n}$ should be acceptable, that is $C L\left(A^{k}\right) \geq \alpha$, i.e.,

$$
C L\left(A^{k}\right)=1-\frac{4}{n(n-1)(n-2)} \sum_{i, j, z=1 ; i<j<z}^{n}\left|a_{i j}^{k}+a_{j z}^{k}-a_{i z}^{k}-0.5\right| \geq \alpha .
$$

Based on the above analysis, the following optimization model is constructed:

$$
\left\{\begin{array}{l}
\min _{A^{k}} k d\left(A^{k}, A^{k, *}\right)=\frac{1}{\left\|K P^{k}\right\|} \sum_{(i, j) \in K P^{k}}\left|a_{i j}^{k}-a_{i j}^{k, *}\right| \\
\text { s.t. }\left\{\begin{array}{l}
C L\left(A^{k}\right)=1-\frac{4}{n(n-1)(n-2)} \sum_{i, j, z=1 ; i<j<z}^{n}\left|a_{i j}^{k}+a_{j z}^{k}-a_{i z}^{k}-0.5\right| \geq \alpha(a) \\
a_{i j}^{k} \in[0,1], \quad i, j=1,2, \ldots, n \\
a_{i j}^{k}+a_{j i}^{k}=1, \quad i, j=1,2, \ldots, n ; i \neq j \\
a_{i i}^{k}=0.5, \quad i=1,2, \ldots, n
\end{array}\right.
\end{array}\right.
$$

Denote model (7) as $P_{3}$. In model $P_{3}, a_{i j}^{k}(i, j=1,2, \ldots, n)$ are the decision variables. The objective function is to minimize the distance between $A^{k, *}=\left(a_{i j}^{k, *}\right)_{n \times n}$ and $A^{k}=\left(a_{i j}^{k}\right)_{n \times n}$ regarding the set $K P^{k}$. Moreover, constraint (a) ensures that the consistency level of $A^{k}$ is acceptable, while constraints (b), (c) and (d) guarantee that $A^{k}$ verifies the properties of Definition 1. Solving model $P_{3}$ will generate the complete APR with acceptable consistency level $A^{k, * *}=\left(a_{i j}^{k, * *}\right)_{n \times n}$. The following proposition provides the equivalent linear programming model to model $P_{3}$.

Proposition 2: $P_{3}$ can be equivalently converted into the following linear programming model (denoted as $P_{4}$ ) via the introduction of some new variables $b_{i j}^{k} \in[0,1] \quad\left((i, j) \in K P^{k}\right)$ and $c_{i j z}^{k} \geq 0$ $(i<j<z ; i, j, z=1, \ldots, n)$ : 


$$
\left\{\begin{array}{l}
\min _{A^{k}} k d\left(A^{k}, A^{k, *}\right)=\frac{1}{\left\|K P^{k}\right\|_{(i, j) \in K P^{k}}} b_{i j}^{k} \\
\text { s.t.t. } \\
\begin{array}{ll}
a_{i j}^{k}-a_{i j}^{k, *} \leq b_{i j}^{k}, \quad(i, j) \in K P^{k} & (a) \\
-a_{i j}^{k}+a_{i j}^{k, *} \leq b_{i j}^{k}, \quad(i, j) \in K P^{k} & (b) \\
a_{i j}^{k}+a_{j z}^{k}-a_{i z}^{k}-0.5 \leq c_{i j z}^{k}, i<j<z ; i, j, z=1, \ldots, n & (c) \\
-a_{i j}^{k}-a_{j z}^{k}+a_{i z}^{k}+0.5 \leq c_{i j z}^{k}, i<j<z ; i, j, z=1, \ldots, n & (d) \\
1-\frac{4}{n(n-1)(n-2)} \sum_{i, j, z=1 ; i<j<z}^{n} c_{i j z}^{k} \geq \alpha & (e) \\
a_{i j}^{k}+a_{j i}^{k}=1, i, j=1,2, \ldots, n ; i \neq j \\
a_{i i}^{k}=0.5, \quad i=1,2, \ldots, n \\
a_{i j}^{k} \in[0,1], i, j=1,2, \ldots, n \\
b_{i j}^{k} \in[0,1], \quad(i, j) \in K P^{k}
\end{array} \\
c_{i j z}^{k} \in[0,1.5], i<j<z ; i, j, z=1, \ldots, n
\end{array}\right.
$$

\section{The proof of Proposition 2 is provided in Appendix B.}

Proposition 2 shows that the optimal complete APR $A^{k, *}=\left(a_{i j}^{k, * *}\right)_{n \times n}$ with an acceptable consistency level of $P_{3}$ can be generated via solving the linear programming model $P_{4}$ with desirable properties listed in Proposition 3 below.

Proposition 3: Let $\dot{A}^{k}=\left(\dot{a}_{i j}^{k}\right)_{n \times n}$ be an incomplete APR.

(i) Recall that $A^{k, *}=\left(a_{i j}^{k, *}\right)_{n \times n}$ is the optimal complete APR associated with $\dot{A}^{k}=\left(\dot{a}_{i j}^{k}\right)_{n \times n}$ derived from model $P_{1}$, and $A^{k, * *}=\left(a_{i j}^{k, * *}\right)_{n \times n}$ is the optimal complete APR associated with $A^{k, *}=\left(a_{i j}^{k, *}\right)_{n \times n}$ derived from model $P_{3}$. Then, it is:

(a) $k d\left(A^{k, * *}, A^{k, *^{*}}\right)=0$ when $C L\left(A^{k, *}\right) \geq \alpha$;

(b) $k d\left(A^{k, * *}, A^{k, *}\right)>0$ when $C L\left(A^{k, *}\right)<\alpha$.

(ii) Let $\alpha_{1}, \alpha_{2} \in[0,1]$ be two consistency thresholds. Let $d^{1, *}$ and $d^{2, *}$ be optimal objective function values of model $P_{3}$ (or $P_{4}$ ) under $\alpha_{1}$ and $\alpha_{2}$, respectively. Then, $d^{1, *} \geq d^{2, *}$ if $\alpha_{1}>\alpha_{2}$

The proof of Proposition 3 is provided in Appendix B.

Proposition 3 shows that if the acceptable consistency level can be achieved by estimating missing preference values in an incomplete APR based on model $P_{1}$, then the preference values in APR $A^{k, *}=\left(a_{i j}^{k, *}\right)_{n \times n}$ regarding the set $K P^{k}$ do not need to be adjusted in $P_{3}$. In addition, Proposition 3 shows that a larger consistency threshold will result in a larger adjustment distance in $P_{3}$. For notation simplicity, $A^{k, *}=\left(a_{i j}^{k, *}\right)_{n \times n}$ and $A^{k, * * *}=\left(a_{i j}^{k, * *}\right)_{n \times n}$ will also be denoted as $A^{k}=\left(a_{i j}^{k}\right)_{n \times n}$. 


\subsection{Personalizing individual semantics}

The PIS of FMEA members means that a linguistic term may have distinct numerical meanings in the LDAMs of different individuals, which is called PNSs. Let $P N S^{k}\left(l_{t}\right)$ be the PNS of the linguistic term $l_{t}(t=0,1, \ldots, g)$ associated with $T M_{k}(k=1,2, \ldots, m)$. In what follows, a deviation minimum-based optimization model to set $P N S^{k}\left(l_{t}\right)(k=1,2, \ldots, m ; t=0,1, \ldots, g)$ to personalize individual semantics of FMEA members in their LDAMs is presented.

(1) Premise to deal with PIS

Based on the PNSs, $P N S^{k}\left(l_{t}\right) \quad(k=1,2, \ldots, m ; t=0,1, \ldots, g)$, the LDAM $U^{k}=\left(u_{i j}^{k}\right)_{n \times y}$ can be converted into the following NAM $V^{k}=\left(v_{i j}^{k}\right)_{n \times y}$ :

$$
v_{i j}^{k}=\sum_{t=0}^{g} \vartheta_{i j, t}^{k} \times P N S^{k}\left(l_{t}\right) .
$$

Premise 1: Because both the NAM $V^{k}$ and the APR $A^{k}$ are associated with the same member $T M_{k}$, their deviation should be as small as possible when setting PNSs.

Therefore, the key task to deal with the PIS issue in the FMEA is to determine PNSs, $P N S^{k}\left(l_{t}\right)$ $(k=1,2, \ldots, m ; t=0,1, \ldots, g)$, that satisfy Premise 1 as described in Fig. 2.

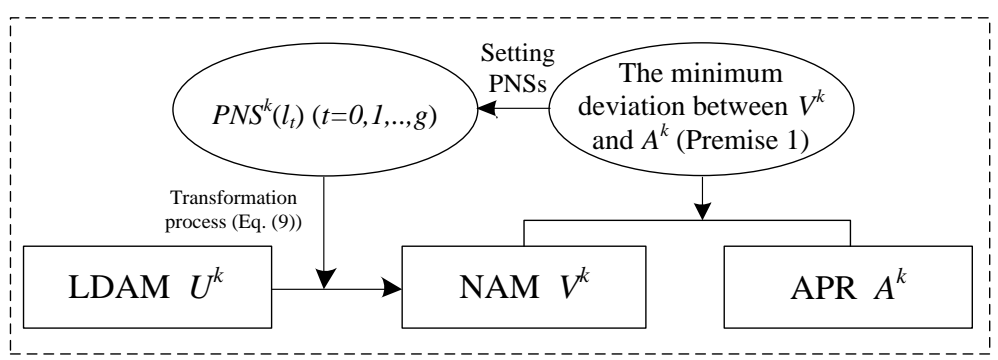

Fig. 2. The basic idea to set $P N S^{k}\left(l_{t}\right) \quad(k=1,2, \ldots, m ; t=0,1, \ldots, g)$

(2) Deviation minimum-based optimization model

Let $P V V^{k}=\left(P V V_{1}^{k}, P V V_{2}^{k}, \ldots, P V V_{n}^{k}\right)^{T}$ be the following preference vector derived from the NAM $V^{k}=\left(v_{i j}^{k}\right)_{n \times y}:$

$$
P V V_{i}^{k}=\frac{B_{i}^{k}}{\sum_{j=1}^{n} B_{j}^{k}},
$$

and

$$
B_{i}^{k}=\sum_{j=0}^{y} \omega_{j} \times v_{i j}^{k}
$$

Let $P V A^{k}=\left(P V A_{1}^{k}, P V A_{2}^{k}, \ldots, P V A_{n}^{k}\right)^{T}$ be the following preference vector derived from the (complete) APR $A^{k}=\left(a_{i j}^{k}\right)_{n \times n}$ :

$$
P V A_{i}^{k}=\frac{2}{n^{2}-n} \sum_{j=1, j \neq i}^{n} a_{i j}^{k} .
$$

When 


$$
P V V_{i}^{k}=\frac{B_{i}^{k}}{\sum_{j=1}^{n} B_{j}^{k}}=P V A_{i}^{k}
$$

we have

$$
B_{i}^{k}=P V A_{i}^{k} \sum_{j=1}^{n} B_{j}^{k}
$$

Therefore, in this paper we use $\sum_{i=1}^{n}\left|B_{i}^{k}-P V A_{i}^{k} \sum_{j=1}^{n} B_{j}^{k}\right|$ to measure the deviation between $P V V^{k}$ and $P V A^{k}$.

As mentioned before, because $V^{k}=\left(v_{i j}^{k}\right)_{n \times y}$ and $A^{k}=\left(a_{i j}^{k}\right)_{n \times n}$ are associated with the same FMEA member $T M_{k}, P V V^{k}=\left(P V V_{1}^{k}, P V V_{2}^{k}, \ldots, P V V_{n}^{k}\right)^{T}$ and $P V A^{k}=\left(P V A_{1}^{k}, P V A_{2}^{k}, \ldots, P V A_{n}^{k}\right)^{T}$ are also both associated with the same FMEA member $T M_{k}$. According to Premise 1, the deviation between $P V V^{k}$ and $P V A^{k}$ should be minimized, i.e.

$$
\min \sum_{i=1}^{n}\left|B_{i}^{k}-P V A_{i}^{k} \sum_{j=1}^{n} B_{j}^{k}\right|
$$

The underlying ordering of the linguistic terms set implies that the PNSs are ordered themselves [17], i.e. $P N S^{k}\left(l_{i}\right)>P N S^{k}\left(l_{j}\right)$ if and only if $i>j$. Moreover, this paper assumes that $P N S^{k}\left(l_{i}\right) \in[(i-1) / g,(i+1) / g], \quad$ and in particular $P N S^{k}\left(l_{0}\right)=0, \quad P N S^{k}\left(l_{g / 2}\right)=0.5 \quad$ and $P N S^{k}\left(l_{g}\right)=1$. In addition, to guarantee the ordering of $P N S^{k}\left(l_{t}\right)$, it is assumed that $P N S^{k}\left(l_{i+1}\right)-P N S^{k}\left(l_{i}\right) \geq \varepsilon$, where $\varepsilon \in(0,3 / g]$ is called the discriminant factor in a PIS model [17-19].

Based on the above analysis, the following deviation minimum-based optimization model is constructed:

$$
\left\{\begin{array}{l}
\min \sum_{i=1}^{n}\left|P V A_{i}^{k} \sum_{j}^{n} B_{j}^{k}-B_{i}^{k}\right| \\
\text { s.t. }\left\{\begin{array}{l}
v_{i j}^{k}=\sum_{t=0}^{g} \vartheta_{i, t}^{k} \times P N S^{k}\left(l_{t}\right), i=1, \ldots, n, j=1, \ldots, y \\
B_{i}^{k}=\sum_{j=0}^{v} w_{j} \times v_{i j}^{k}, i=1, \ldots, n \\
P V A_{i}^{k}=\frac{2}{n^{2}-n} \sum_{j=1, j \neq i}^{n} a_{i j}^{k}, i=1, \ldots, n \\
P N S^{k}\left(l_{0}\right)=0 \\
P N S^{k}\left(l_{g / 2}\right)=0.5 \\
P N S^{k}\left(l_{g}\right)=1 \\
P N S^{k}\left(l_{i+1}\right)-P N S^{k}\left(l_{i}\right) \geq \varepsilon, i=0, \ldots, g-1 \\
P N S^{k}\left(l_{i}\right) \in[(i-1) / g,(i+1) / g], i=1, \ldots, g-1
\end{array}\right.
\end{array}\right.
$$

Denote model (16) as $P_{5} . P N S^{k}\left(l_{t}\right) \quad(t=0,1, \ldots, g)$ are the decision variables of model $P_{5}$. By solving model $P_{5}$, optimal values of $P N S^{k}\left(l_{t}\right) \quad(t=0,1, \ldots, g)$ are obtained: $P N S^{k, *}\left(l_{t}\right)$. Based on $P N S^{k, *}\left(l_{t}\right)$, the optimal NAM $V^{k, *}=\left(v_{i j}^{k, *}\right)_{n \times y}$ associated with $V^{k}=\left(v_{i j}^{k}\right)_{n \times y}$ is computed as per expression (9): $v_{i j}^{k, *}=\sum_{t=0}^{g} \vartheta_{i j, t}^{k} \times P N S^{k, *}\left(l_{t}\right)$. Furthermore, as per expressions (10) and (11) the preference vector $\quad P V V^{k, *}=\left(P V V_{1}^{k, *}, P V V_{2}^{k, *}, \ldots, P V V_{n}^{k, *}\right)^{T} \quad$ associated with $\quad V^{k, *}=\left(v_{i j}^{k, *}\right)_{n \times y} \quad$ is 
obtained: $\quad P V V_{i}^{k, *}=\sum_{j=0}^{y} \omega_{j} \times v_{i j}^{k, *} / \sum_{h=1}^{n} \sum_{j=0}^{y} \omega_{j} \times v_{h j}^{k, *}$. For notation simplicity, $P N S^{k, *}\left(l_{t}\right)$, $V^{k, *}=\left(v_{i j}^{k, *}\right)_{n \times y}$, and $P V V^{k, *}$ will also be denoted as $P N S^{k}\left(l_{t}\right), V^{k}=\left(v_{i j}^{k}\right)_{n \times y}$, and $P V V^{k}$, respectively.

In model $P_{5}$, changing the objective function to $\min \sum_{i=1}^{n} c_{i}^{k}$ and adding the following two constraints $P V A_{i}^{k} \sum_{j}^{n} B_{j}^{k}-B_{i}^{k} \leq c_{i}^{k}$ and $-P V A_{i}^{k} \sum_{j}^{n} B_{j}^{k}+B_{i}^{k} \leq c_{i}^{k} \quad\left(c_{i}^{k} \geq 0, i=1, \ldots, n\right)$ results in a new linear optimization model, which is denoted as $P_{6}$. Similar to Propositions 1 and 2 , it can be proved that models $P_{5}$ and $P_{6}$ are equivalent, which implies that the optimal solutions of $\operatorname{PNSs,} \operatorname{PNS}^{k}\left(l_{t}\right)$ $(t=0,1, \ldots, g)$, of model $P_{5}$ can be obtained by solving the linear programming model $P_{6}$.

Let $R V^{k}=\left(R V_{1}^{k}, R V_{2}^{k}, \ldots, R V_{n}^{k}\right)^{T}$ be the risk ordering of failure modes obtained from $P V V^{k}=\left(P V V_{1}^{k}, P V V_{2}^{k}, \ldots, P V V_{n}^{k}\right)^{T}$, where $R V_{i}^{k}=j$ if $P V V_{i}^{k}$ is the $j$ th highest value in $\left\{P V V_{1}^{k}, P V V_{2}^{k}, \ldots, P V V_{n}^{k}\right\}$. Analogously, we obtain the risk ordering $R A^{k}=\left(R A_{1}^{k}, R A_{2}^{k}, \ldots, R A_{n}^{k}\right)^{T}$ of failure modes from $P V A^{k}=\left(P V A_{1}^{k}, P V A_{2}^{k}, \ldots, P V A_{n}^{k}\right)^{T}$. The following proposition guarantees that the two risk orderings derived from $V^{k}$ (or $U^{k}$ ) and $A^{k}$ coincide when $\sum_{i=1}^{n}\left|B_{i}^{k}-P V A_{i}^{k} \sum_{j=1}^{n} B_{j}^{k}\right|=0$.

Proposition 4: If $\sum_{i=1}^{n}\left|B_{i}^{k}-P V A_{i}^{k} \sum_{j=1}^{n} B_{j}^{k}\right|=0$, then $R V^{k}=R A^{k}$.

The proof of Proposition 4 is provided in Appendix B.

\subsection{Failure mode ranking}

The risk ordering of failure modes $F M=\left\{F M_{1}, F M_{2}, \ldots, F M_{n}\right\}$ is obtained from the preference vectors $\left\{P V V^{1}, P V V^{2}, \ldots, P V V^{m}\right\}$ and $\left\{P V A^{1}, P V A^{2}, \ldots, P V A^{m}\right\}$. Specifically, let $P V V^{c}=\left(P V V_{1}^{c}, P V V_{2}^{c}, \ldots, P V V_{n}^{c}\right)^{T}$ and $P V A^{c}=\left(P V A_{1}^{c}, P V A_{2}^{c}, \ldots, P V A_{n}^{c}\right)^{T}$ be the following collective preference vectors derived from $\left\{P V V^{1}, P V V^{2}, \ldots, P V V^{m}\right\}$ and $\left\{P V A^{1}, P V A^{2}, \ldots, P V A^{m}\right\}$, respectively:

$$
\begin{aligned}
& P V V_{i}^{c}=\sum_{k=1}^{m} \lambda_{k} \cdot P V V_{i}^{k}, \\
& P V A_{i}^{c}=\sum_{k=1}^{m} \lambda_{k} \cdot P V A_{i}^{k} .
\end{aligned}
$$

Let the overall preference vector over failure modes $E^{c}=\left(E_{1}^{c}, E_{2}^{c}, \ldots, E_{n}^{c}\right)^{T}$ be the following linear combination of the above two collective preference vectors:

$$
E_{i}^{c}=\tau \cdot P V V_{i}^{c}+(1-\tau) \cdot P V A_{i}^{c}
$$

where $\tau \in[0,1]$ is a parameter that should be set based on the actual FMEA situation. The collective risk ordering $R^{c}=\left(r_{1}^{c}, r_{2}^{c}, \ldots, r_{n}^{c}\right)^{T}$ of failure modes $F M=\left\{F M_{1}, F M_{2}, \ldots, F M_{n}\right\}$ is generated using their corresponding overall preference values in $E^{c}$.

Formally, we use the following algorithm (Algorithm I) to describe the PIS-based linguistic FMEA approach. 
Table 1: Algorithm I

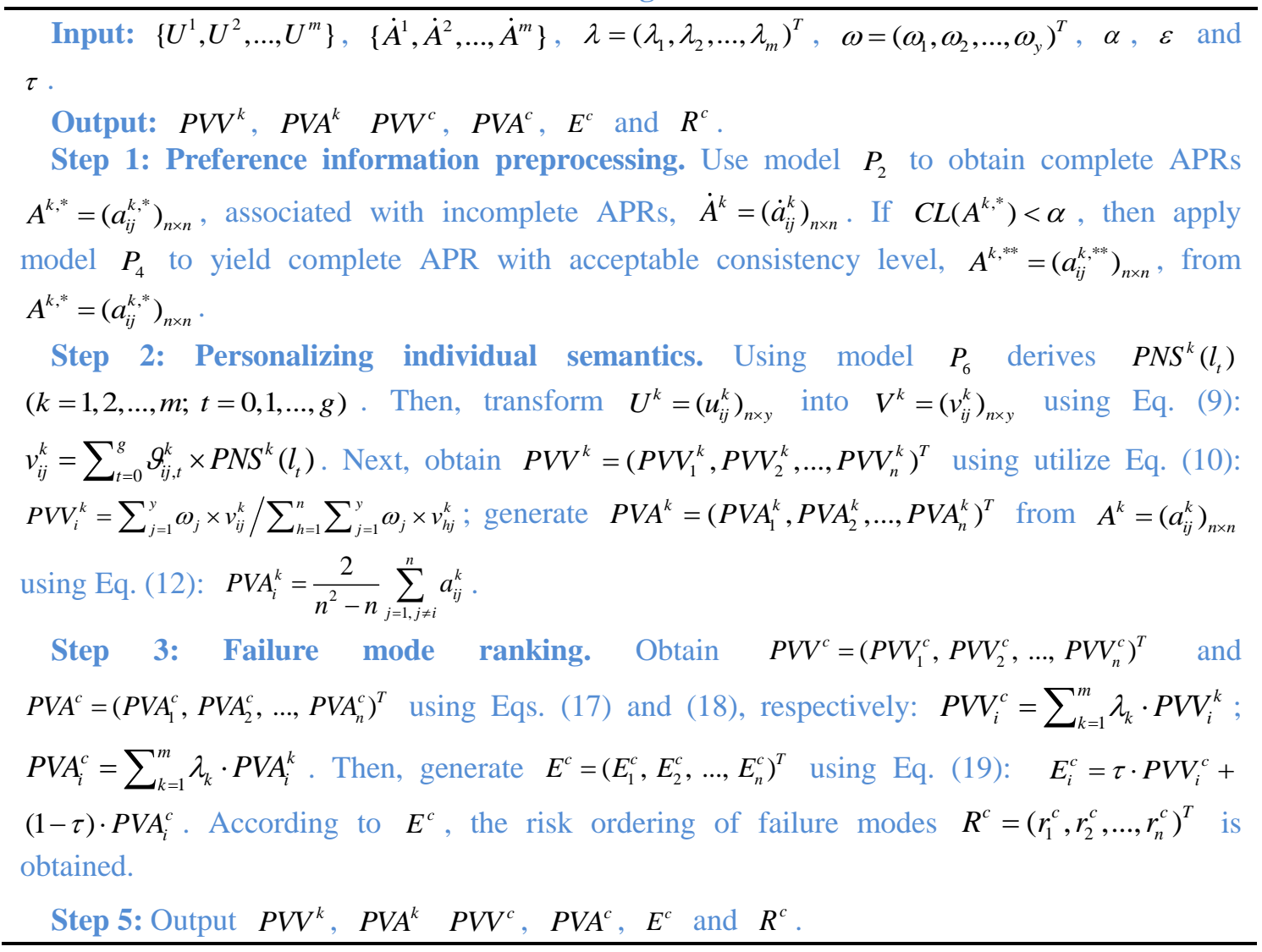

\section{Case study}

This section shows the practical use of the PIS-based linguistic FMEA approach to the problem of the reliability management of blood transfusion [25, 27]. For the sake of clarity and readability, some data and materials in the case study are in Appendix C.

\subsection{Problem description and data collection}

A blood transfusion for a person who is badly injured or ill is a common clinical diagnosis and treatment method in healthcare organizations and, at the same time, a costly and complex process. Risks of a blood transfusion process, which can cause great damage to the patient, include: hemolytic reactions, spreading infectious diseases and allergic reaction. Thus, identifying and eliminating potential failures or risks is critical to the safety and reliability of the blood transfusion, and the PIS-based linguistic FMEA approach is therefore suitable to manage the reliability of blood transfusion when FMEA members express their assessments in linguistic terms.

In $\mathrm{Lu}$ et al. [27], nineteen potential failure modes were initially identified in the whole process of blood transfusion. In the case study, four failure modes $\left\{F M_{1}, F M_{2}, F M_{3}, F M_{4}\right\}$ with high risk levels are chosen for further detailed illustration. The four failure modes and their causes and effects are provided (see Table A1 in Appendix C). 
As analyzed in Section 1, the risk assessment and prioritization of failure modes is an essential issue in the FMEA. To conduct this task, a total of sixteen eligible members $\left\{T M_{1}, T M_{2}, \ldots, T M_{16}\right\}$ are invited to conduct the risk assessment. They are equally important, i.e. the weight vector of the sixteen members is $\lambda=(1 / 16,1 / 16, \ldots, 1 / 16)^{T}$. The sixteen members assess the four failure modes based on the three risk factors of $O$ (occurrence), $S$ (severity), and $D$ (detection), which are denoted herein $R F_{1}=O, R F_{2}=S$, and $R F_{3}=D$, and they are assumed to be equally important, i.e. the weight vector of the three risk factors is $\omega=(1 / 3,1 / 3,1 / 3)^{T}$.

Due to the complexity and uncertainty of the reliability management of the blood transfusion, the sixteen FMEA members provide their opinions on the four identified failure modes using the linguistic distribution assessment approach. In particular, the seven-grade linguistic terms set $L$ (see Table A2 in Appendix C) is used by the sixteen FMEA members to rate failure modes. When a member expresses preferences based on the linguistic distribution assessment of $L$, he/she will directly assign symbolic proportions to the linguistic terms in $L$. The LDAMs, $\left\{U^{1}, U^{2}, \ldots, U^{16}\right\}$, provided by the sixteen FMEA members are in Table A3 in Appendix C. Meanwhile, the sixteen FMEA members provide their incomplete APRs over the four failure modes $\left\{\dot{A}^{1}, \dot{A}^{2}, \ldots, \dot{A}^{16}\right\}$, which are in Appendix C.

The different experiences, cultures and educational backgrounds of the sixteen FMEA members imply the existence of PIS in their provided LDAMs. The problem is concerned with setting PNSs to personalize individual semantics in FMEA members' LADMs $\left\{U^{1}, U^{2}, \ldots, U^{16}\right\}$, and then finding a risk ordering of the four failure modes $\left\{F M_{1}, F M_{2}, F M_{3}, F M_{4}\right\}$ based on $\left\{U^{1}, U^{2}, \ldots, U^{16}\right\}$, $\left\{\dot{A}^{1}, \dot{A}^{2}, \ldots, \dot{A}^{16}\right\}$ and the PNSs. In this example, the parameter values are set as $\alpha=0.9$ and $\varepsilon=0.05$, respectively. It should be noted that the PIS-based linguistic FMEA approach is still useful with the setting of different parameter values to the above ones.

\subsection{Illustration of the PIS-based linguistic FMEA approach}

The described three-step procedure is implemented in the PIS-based linguistic FMEA approach.

\section{Step 1: Preference information preprocessing}

Using the two-stage optimization model $\left(P_{1}\right.$ and $\left.P_{3}\right)$ presented in Section 4.1, the incomplete APRs $\left\{\dot{A}^{1}, \dot{A}^{2}, \ldots, \dot{A}^{16}\right\}$ are transformed into complete APRs with acceptable consistency levels $\left\{A^{1}, A^{2}, \ldots, A^{16}\right\}$. Here, we illustrate the process of obtaining $A^{1}$ from $\dot{A}^{1}$.

(i) Application of the consistency maximum-based optimization model $\left(P_{1}\right)$ to estimate missing values in $\dot{A}^{1}$

Take $\dot{A}^{1}$ as the input of $P_{1}$, the following model is presented: 


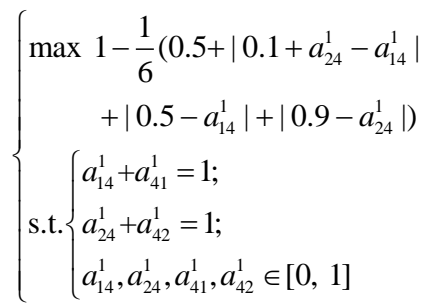

From Proposition 1, this model is transformed into its equivalent linear programing model $P_{2}$ with optimal solution values $a_{14}^{1, *}=0.7, a_{24}^{1, *}=0.9, a_{41}^{1, *}=0.3$, and $a_{42}^{1, * *}=0.1$. Thus, the optimal complete APR $A^{1, *}$ :

$$
A^{1, * *}=\left(\begin{array}{cccc}
0.5 & 0.6 & 0.4 & 0.7 \\
0.4 & 0.5 & 0.8 & 0.9 \\
0.1 & 0.2 & 0.5 & 0.6 \\
0.3 & 0.1 & 0.4 & 0.5
\end{array}\right)
$$

According to Eq. (1), we obtain that $C L\left(A^{1, *}\right)=0.8333$ and, because $C L\left(A^{1, *}\right)=0.8333<\alpha=0.9$, model $P_{3}$ is applied to improve the consistency level of $A^{1, *}$.

(ii) Application of the adjustment minimum-based optimization model ( $P_{3}$ ) for consistency improvement for $A^{1, *}$

Take $A^{1, *}$ as the input of $P_{3}$, the following model is generated:

$$
\left\{\begin{array}{l}
\min \frac{1}{8}\left(\left|0.6-a_{12}^{1}\right|+\left|0.4-a_{13}^{1}\right|+\left|0.8-a_{23}^{1}\right|+\left|0.6-a_{34}^{1}\right|\right. \\
\left.\quad+\left|0.4-a_{21}^{1}\right|+\left|0.1-a_{31}^{1}\right|+\left|0.2-a_{32}^{1}\right|+\left|0.4-a_{43}^{1}\right|\right) \\
\text { s.t. }\left\{\begin{array}{l}
1-\frac{1}{6}\left(\left|a_{12}^{1}+a_{23}^{1}-a_{13}^{1}-0.5\right|+\left|a_{12}^{1}+a_{24}^{1}-a_{14}^{1}-0.5\right|\right. \\
\left.+\left|a_{13}^{1}+a_{34}^{1}-a_{14}^{1}-0.5\right|+\left|a_{23}^{1}+a_{34}^{1}-a_{24}^{1}-0.5\right|\right) \geq 0.9 ; \\
a_{12}^{1}+a_{21}^{1}=1 ; a_{13}^{1}+a_{31}^{1}=1 ; a_{14}^{1}+a_{41}^{1}=1 ; a_{23}^{1}+a_{32}^{1}=1 ; a_{24}^{1}+a_{42}^{1}=1 ; a_{34}^{1}+a_{43}^{1}=1 ; \\
a_{11}^{1}=0.5 ; a_{22}^{1}=0.5 ; a_{33}^{1}=0.5 ; a_{44}^{1}=0.5 ; \\
a_{12}^{1}, a_{13}^{1}, a_{14}^{1}, a_{21}^{1}, a_{23}^{1}, a_{24}^{1}, a_{31}^{1}, a_{32}^{1}, a_{34}^{1}, a_{41}^{1}, a_{42}^{1}, a_{43}^{1}, \in[0,1]
\end{array}\right.
\end{array}\right.
$$

From Proposition 2, the above model can be converted into an equivalent linear programing model with solution APR with acceptable consistency level:

$$
A^{1, * \text {, }}=\left(\begin{array}{cccc}
0.5 & 0.5406 & 0.4499 & 0.6293 \\
0.4594 & 0.5 & 0.7093 & 0.6831 \\
0.5501 & 0.2907 & 0.5 & 0.6 \\
0.3707 & 0.3169 & 0.4 & 0.5
\end{array}\right) .
$$

For convenience, $A^{1, * *}$ is denoted as $A^{1}$ in our study. The same process used to obtain $A^{1}$ is repeated to derive complete APRs with acceptable consistency levels $\left\{A^{2}, A^{3}, \ldots, A^{16}\right\}$ associated with $\left\{\dot{A}^{2}, \dot{A}^{3}, \ldots, \dot{A}^{16}\right\}$, which are listed in Appendix C.

\section{Step 2: Personalizing individual semantics}

In this step, the deviation minimum-based optimization model $\left(P_{5}\right)$ described in Section 4.2 is used to obtain PNSs of linguistic terms associated with the sixteen FMEA members. Here, we show the process to obtain $P N S^{1}\left(l_{t}\right) \quad(t=0,1, \ldots, 6)$. Taking $A^{1}$ and $U^{1}$ as the input of model $P_{5}$ yields: 


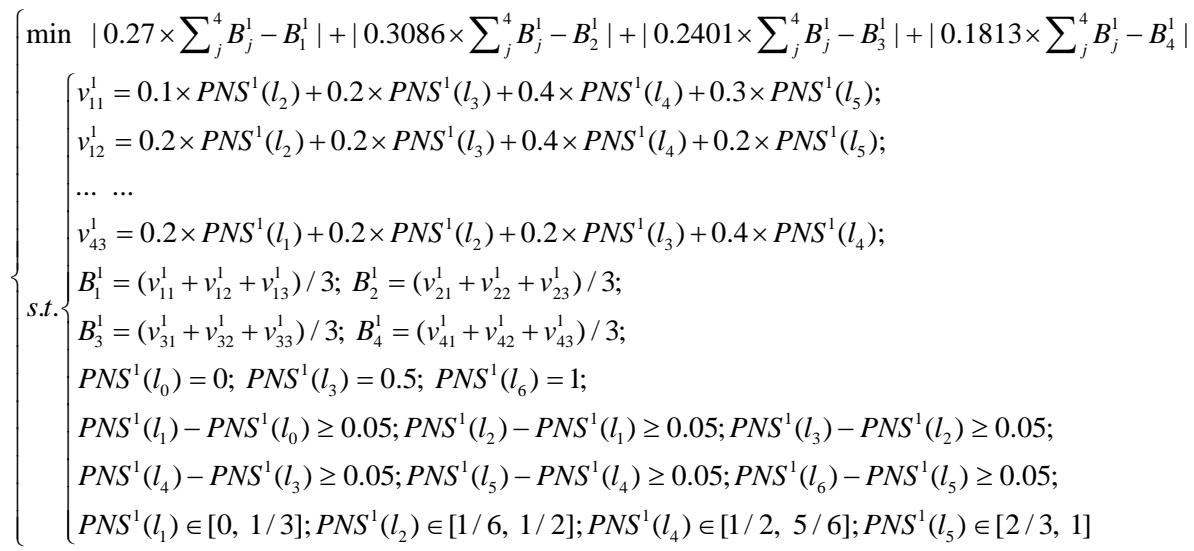

Similarly to the transformation process of model $P_{1}$ into model $P_{2}$, values for $\operatorname{PNS}^{1}\left(l_{t}\right)$ $(t=0,1, \ldots, 6)$ are obtained by transforming model $P_{5}$ into an equivalent linear programming model. The same process is repeated to obtain the values of $P N S^{k}\left(l_{t}\right) \quad(k=2,3, \ldots, 16 ; t=0,1, \ldots, 6)$, which are provided in Table 2.

Table 2: PNSs of linguistic terms for each FMEA member

\begin{tabular}{lccccccc}
\hline & $P N S\left(l_{0}\right)$ & $P N S\left(l_{1}\right)$ & $P N S\left(l_{2}\right)$ & $P N S\left(l_{3}\right)$ & $P N S\left(l_{4}\right)$ & $P N S\left(l_{5}\right)$ & $P N S\left(l_{6}\right)$ \\
\cline { 2 - 7 }$T M_{1}$ & 0 & 0.05 & 0.45 & 0.5 & 0.55 & 0.6801 & 1 \\
$T M_{2}$ & 0 & 0.05 & 0.1667 & 0.5 & 0.8333 & 0.95 & 1 \\
$T M_{3}$ & 0 & 0.3333 & 0.3833 & 0.5 & 0.55 & 0.95 & 1 \\
$T M_{4}$ & 0 & 0.2776 & 0.3276 & 0.5 & 0.55 & 0.6667 & 1 \\
$T M_{5}$ & 0 & 0.3333 & 0.4241 & 0.5 & 0.6167 & 0.6667 & 1 \\
$T M_{6}$ & 0 & 0.05 & 0.1667 & 0.5 & 0.5548 & 0.95 & 1 \\
$T M_{7}$ & 0 & 0.05 & 0.1667 & 0.5 & 0.55 & 0.6667 & 1 \\
$T M_{8}$ & 0 & 0.1616 & 0.2116 & 0.5 & 0.55 & 0.6667 & 1 \\
$T M_{9}$ & 0 & 0.0593 & 0.45 & 0.5 & 0.55 & 0.95 & 1 \\
$T M_{10}$ & 0 & 0.2838 & 0.3338 & 0.5 & 0.55 & 0.6983 & 1 \\
$T M_{11}$ & 0 & 0.05 & 0.1667 & 0.5 & 0.55 & 0.6667 & 1 \\
$T M_{12}$ & 0 & 0.2029 & 0.2529 & 0.5 & 0.6167 & 0.6667 & 1 \\
$T M_{13}$ & 0 & 0.3106 & 0.45 & 0.5 & 0.55 & 0.6667 & 1 \\
$T M_{14}$ & 0 & 0.1544 & 0.2044 & 0.5 & 0.55 & 0.6667 & 1 \\
$T M_{15}$ & 0 & 0.05 & 0.1667 & 0.5 & 0.833 & 0.9482 & 1 \\
$T M_{16}$ & 0 & 0.3333 & 0.45 & 0.5 & 0.5514 & 0.6667 & 1
\end{tabular}

Meanwhile, based on the PNSs values, via Eq. (9) the LDAMs are transformed into NAMs, $V^{k}=\left(v_{i j}^{k}\right)_{4 \times 3} \quad(k=1,2, \ldots, 16) \quad$ (see Table A4 in Appendix C), while Eq. (10) is used to obtain $P V V^{k}=\left(P V V_{1}^{k}, P V V_{2}^{k}, P V V_{3}^{k}, P V V_{4}^{k}\right)^{T}$ from $V^{k}=\left(v_{i j}^{k}\right)_{4 \times 3}$ and Eq. (12) to obtain $P V A^{k}=\left(P V A_{1}^{k}\right.$, $\left.P V A_{2}^{k}, P V A_{3}^{k}, P V A_{4}^{k}\right)^{T}$ from $A^{k}=\left(a_{i j}^{k}\right)_{4 \times 4} \quad(k=1,2, \ldots, 16)$, which are provided in Appendix C.

\section{Step 3: Failure mode ranking}

Eqs. (17) and (18) are applied to compute $P V V^{c}=\left(P V V_{1}^{c}, P V V_{2}^{c}, P V V_{3}^{c}, P V V_{4}^{c}\right)^{T}$ : $P V V_{i}^{c}=\left(P V V_{i}^{1}+P V V_{i}^{2}+\ldots+P V V_{i}^{16}\right) / 16 \quad(i=1,2,3,4) ;$ and $P V A^{c}=\left(P V A_{1}^{c}, P V V_{2}^{c}, P V V_{3}^{c}, P V V_{4}^{c}\right)^{T}:$ $P V A_{i}^{c}=\left(P V A_{i}^{1}+P V A_{i}^{2}+\ldots+P V A_{i}^{16}\right) / 16(i=1,2,3,4)$, respectively. 
$P V V^{c}=(0.2737,0.2740,0.2348,0.2175)^{T}$, $P V A^{c}=(0.2788,0.2752,0.2414,0.2046)^{T}$.

Setting $\tau=0.5$, Eq. (19) becomes $E^{c}=\left(E_{1}^{c}, E_{2}^{c}, E_{3}^{c}, E_{4}^{c}\right)^{T}: E_{i}^{c}=0.5 \times P V V_{i}^{c}+0.5 \times P V A_{i}^{c}(i=1,2,3,4)$.

$$
E^{c}=(0.2763,0.2746,0.2381,0.2110)^{T} .
$$

Based on $E^{c}$, the risk ordering of the four failure modes is $R^{c}=(1,2,3,4)^{T}$ (i.e., $\left.F M_{1} \succ F M_{2} \succ F M_{3} \succ F M_{4}\right)$. Therefore, failure mode $F M_{1}$ should be paid more attention for risk mitigation.

\section{Comparison analysis}

This section presents several criteria and designs simulation methods to compare the performances of the proposed PIS-based linguistic FMEA approach and the FNS-based linguistic FMEA approach, which is not a PIS based approach.

\subsection{Comparison criteria}

In the PIS-based linguistic FMEA approach, there are two kinds of assessment information: LDAMs and APRs. Recall from Section 4.2 that $P V V^{k}=\left(P V V_{1}^{k}, P V V_{2}^{k}, \ldots, P V V_{n}^{k}\right)^{T}$ and $P V A^{k}=\left(P V A_{1}^{k}, P V A_{2}^{k}, \ldots, P V A_{n}^{k}\right)^{T}$ are the preference vectors associated with LDAM $U^{k}=\left(u_{i j}^{k}\right)_{n \times y}$ and APR $A^{k}=\left(a_{i j}^{k}\right)_{n \times n}$, respectively; $R V^{k}=\left(R V_{1}^{k}, R V_{2}^{k}, \ldots, R V_{n}^{k}\right)^{T}$ and $R A^{k}=\left(R A_{1}^{k}, R A_{2}^{k}, \ldots, R A_{n}^{k}\right)^{T}$ are the risk orderings of failure modes obtained from $P V V^{k}$ and $P V A^{k}$, respectively.

Both preference vectors $P V V^{k}$ and $P V A^{k}$ are associated with the same FMEA member $T_{M_{k}}$, so it is natural that the preference vectors $P V V^{k}$ and $P V A^{k}$ should be as consistent as possible, which is also expected to be the case between the risk orderings $R V^{k}$ and $R A^{k}$. Therefore, in this paper the performance of the proposed PIS-based linguistic FMEA approach is evaluated by measuring the deviation between $P V V^{k}$ and $P V A^{k}$, and the deviation between $R V^{k}$ and $R A^{k}$. The Manhattan distance and the Euclidean distance have been widely used to measure the deviation between preference vectors (or risk orderings), and thus we propose the following four comparison criteria.

(1) The Manhattan distance between preference vectors $P V V^{k}$ and $P V A^{k}$ :

$$
M P^{k}=\sum_{i=1}^{n}\left|P V V_{i}^{k}-P V A_{i}^{k}\right|
$$

(2) The Euclidean distance between the preference vectors $P V V^{k}$ and $P V A^{k}$ :

$$
E P^{k}=\sqrt{\sum_{i=1}^{n}\left(P V V_{i}^{k}-P V A_{i}^{k}\right)^{2}}
$$

(3) The Manhattan distance between the risk orderings $R V^{k}$ and $R A^{k}$ :

$$
M R^{k}=\sum_{i=1}^{n}\left|R V_{i}^{k}-R A_{i}^{k}\right|
$$

(4) The Euclidean distance between risk orderings $R V^{k}$ and $R A^{k}$ :

$$
E R^{k}=\sqrt{\sum_{i=1}^{n}\left(R V_{i}^{k}-R A_{i}^{k}\right)^{2}}
$$


The smaller the values $M P^{k}$ and $E P^{k}$ are, the better the consistency between the preference vectors $P V V^{k}$ and $P V A^{k}$ are. The smaller the values $M R^{k}$ and $E R^{k}$ are, the better the consistency between $R V^{k}$ and $R A^{k}$ are. So, smaller values of $\boldsymbol{M} \boldsymbol{P}^{k}, E P^{k}, \boldsymbol{M R ^ { k }}$ and $E R^{k}$ indicate better performance of linguistic FMEA approaches.

\subsection{Comparison methods and results}

The FNS-based linguistic FMEA approach does not consider the PIS issue (e.g., [14, 24, 34]), because it is based on the fixed numerical scale function $N S^{k}\left(l_{i}\right)=i / g \quad(k=1,2, \ldots, m ; i=0,1, \ldots, g)$. Clearly, the FNS-based linguistic FMEA approach can be considered a special case of PIS-based linguistic FMEA approach with $P N S^{k}\left(l_{i}\right)=i / g(k=1,2, \ldots, m ; i=0,1, \ldots, g)$. Thus, a minor revision of Algorithm I is needed to create Algorithm II (see Appendix D) to describe the FNS-based linguistic FMEA approach within the proposed FNS-based linguistic FMEA framework.

In the following, we compare the PIS and FNS based linguistic FMEA approaches.

(1) Comparison using the case study data

Using the data from the case study of Section 5, we obtain values for $M P^{k}, E P^{k}, M R^{k}$, and $E R^{k} \quad(k=1,2, \ldots, 16)$ under the PIS and FNS based linguistic FMEA approaches, which are plotted in Fig. 3.

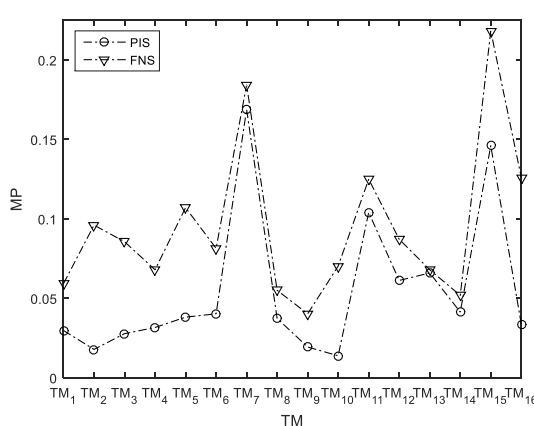

(a) MP

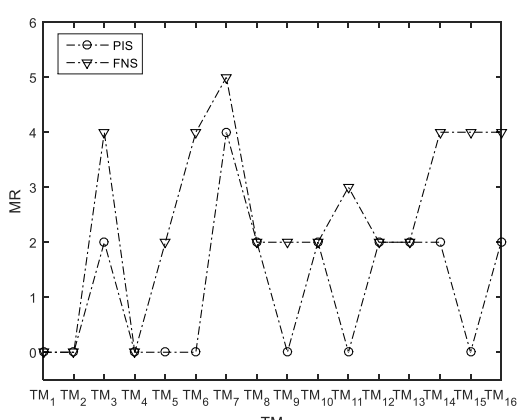

(c) MR

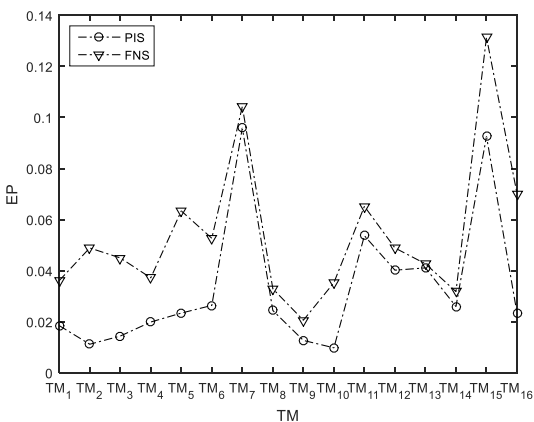

(b) $\mathrm{EP}$

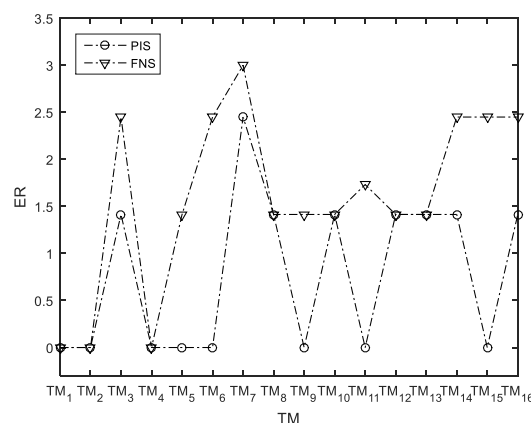

(d) ER

Fig. 3. Comparison results based on the case study data

Fig. 3 shows that the values for $M P^{k}, E P^{k}, M R^{k}$ and $E R^{k}$ obtained with the PIS-based linguistic FMEA approach are lower than or equal to those obtained with the FNS-based linguistic FMEA approach for all sixteen FMEA members. Thus, the PIS-based linguistic FMEA approach performs better than the FNS-based linguistic FMEA approach in the four considered criteria. 
(2) Comparison with random data

In order to obtain compelling results, Simulation methods I and II with randomly generated data are designed to compare the PIS and FNS based linguistic FMEA approaches.

The basic idea of Simulation methods I and II (see Table A5 in Appendix D) is to randomly generate the LDAMs and APRs associated with $m$ FMEA members, and then use the generated data in Algorithm I (PIS-based linguistic FMEA approach) and in Algorithm II (FNS-based linguistic FMEA approach) to generate $P V V^{k}$ and $P V A^{k} \quad(k=1,2, \ldots, m)$, and eventually to obtain the values of $M P^{k}, E P^{k}, M R^{k}$, and $E R^{k} \quad(k=1,2, \ldots, m)$ under the PIS and FNS based linguistic FMEA approaches, respectively. Furthermore, under the PIS and FNS based linguistic FMEA approaches the average values of $\left\{M P^{1}, \ldots, M P^{m}\right\},\left\{E P^{1}, \ldots, E P^{m}\right\},\left\{M R^{1}, \ldots, M R^{m}\right\}$ and $\left\{E R^{1}, \ldots, E R^{m}\right\}$ are proposed to compare performance, with smaller values of $A M P, A E P, A M R$ and $A E R$ indicating better performance of the linguistic FMEA approach:

$$
\begin{gathered}
A M P=\frac{1}{m} \sum_{k=1}^{m} M P^{k} \\
A E P=\frac{1}{m} \sum_{k=1}^{m} E P^{k} \\
A M R=\frac{1}{m} \sum_{k=1}^{m} M R^{k} \\
A E R=\frac{1}{m} \sum_{k=1}^{m} E R^{k}
\end{gathered}
$$

Notably, complete APRs with acceptable consistency are randomly generated to use in Simulation methods I and II to better analyze the effect of PIS on the reliability management quality.

Without loss of generality, the following parameter values are set in Simulation methods I and II: $\tau=0.5, \quad y=3$ and $\lambda=\left(\frac{1}{m}, \frac{1}{m}, \ldots, \frac{1}{m}\right)^{T}$. Moreover, the following three combination of parameters scenarios are considered: (i) $m=9, n=3, g=6, \varepsilon=0.01, \alpha=\{0.8,0.82, \ldots, 0.9\}$ and $\omega=(0.7,0.2,0.1)^{T} ;$ (ii) $m=7, \quad n=4, \quad g=6, \varepsilon=0.01, \quad \alpha=\{0.8,0.82, \ldots, 0.9\} \quad$ and $\omega=(0.15,0.55,0.3)^{T} ;$ (iii) $m=5, \quad n=5, \quad g=8, \quad \varepsilon=0.005, \quad \alpha=\{0.8,0.82, \ldots, 0.9\}$ and $\omega=(0.1,0.1,0.8)^{T}$.

Then, Simulation methods I and II are run 1000 times for each one of the three different parameters scenarios to obtain the average values of $A M P, A E P, A M R$, and $A E R$ for both the PIS and FNS based linguistic FMEA approaches, respectively, and plotted in Figs. 4-6. Clearly, the average values of $A M P$ and $A E P, A M R$ and $A E R$ under the PIS-based linguistic FMEA approach are smaller than those under the FNS-based linguistic FMEA approach in the three parameter scenarios, which is again consistent with the results obtained using the case study data. These findings show that taking the PIS issue into account the PIS-based linguistic FMEA approach can improve the reliability management quality. 


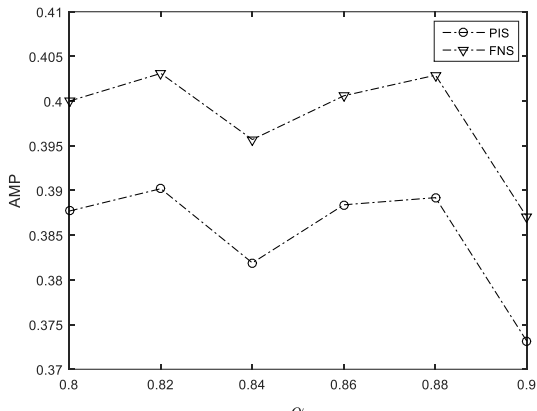

(a) AMP

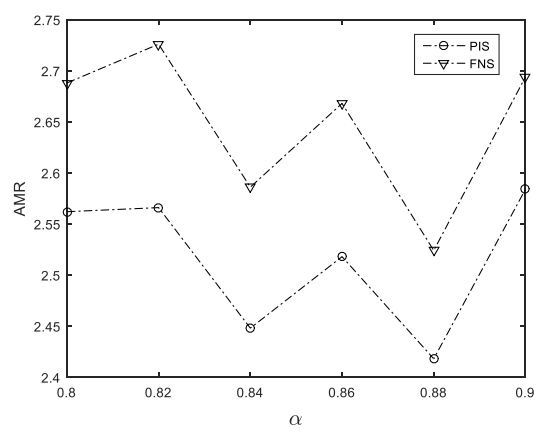

(c) AMR

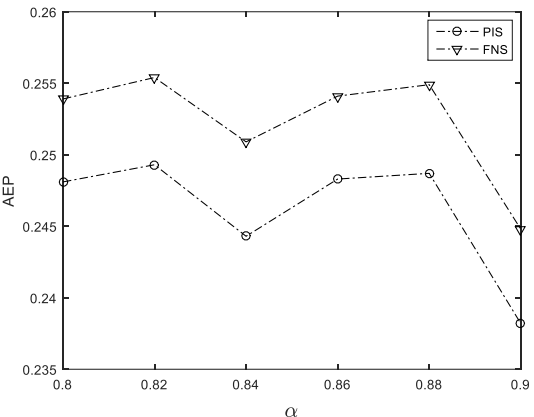

(b) AEP

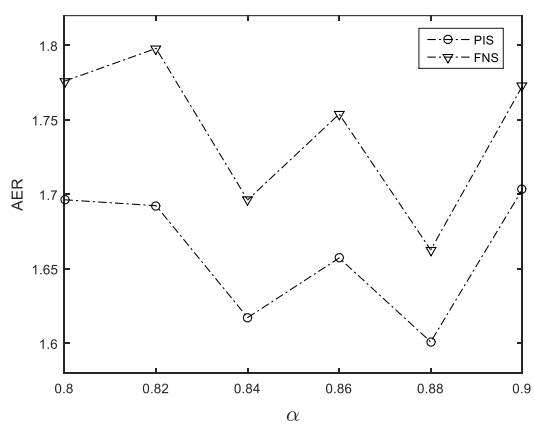

(d) AER

Fig. 4. Comparison results of Simulation methods I and II in parameters scenario (i)

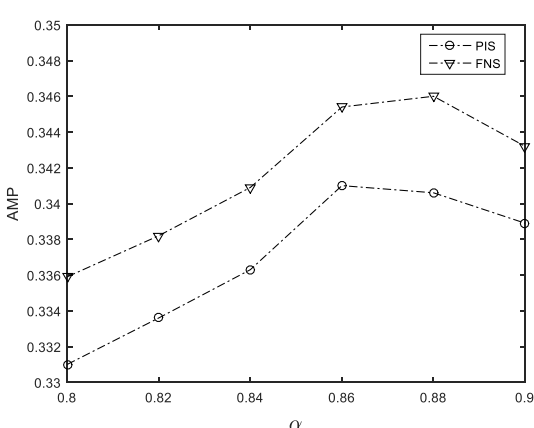

(a) AMP

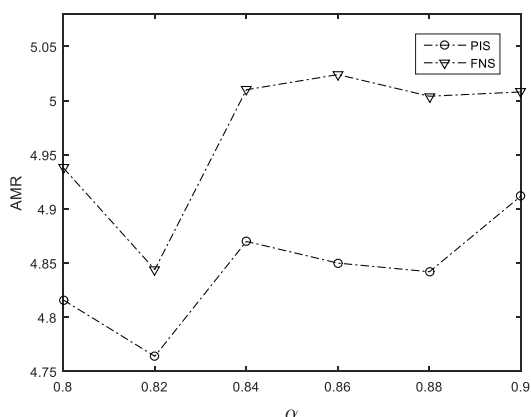

(c) AMR

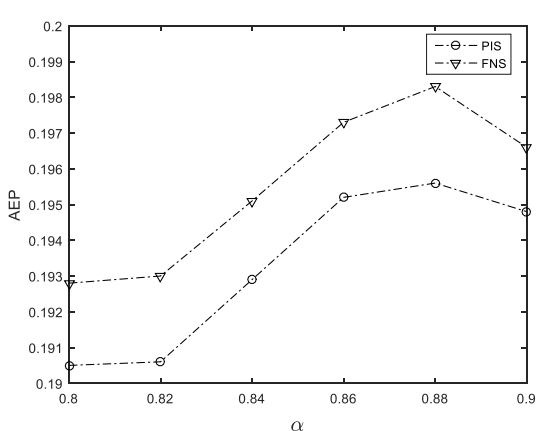

(b) AEP

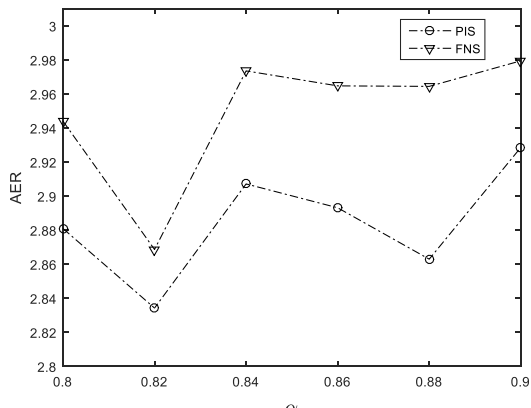

(d) AER

Fig. 5. Comparison results of Simulation methods I and II in parameters scenario (ii) 


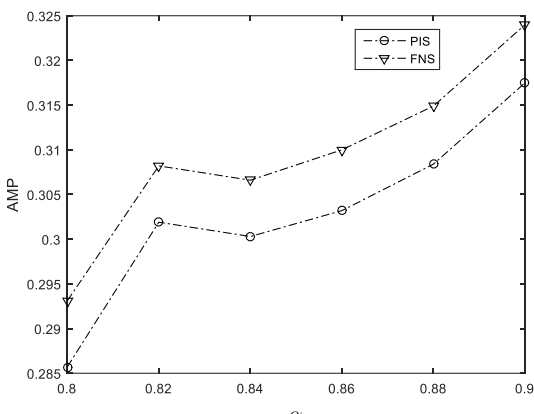

(a) AMP

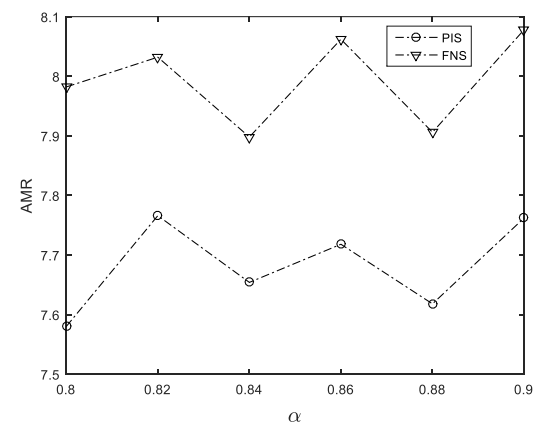

(c) AMR

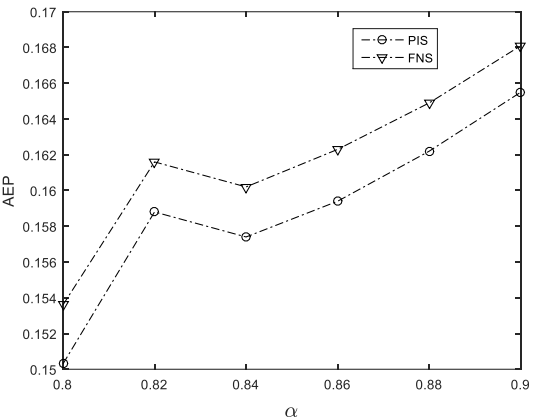

(b) AEP

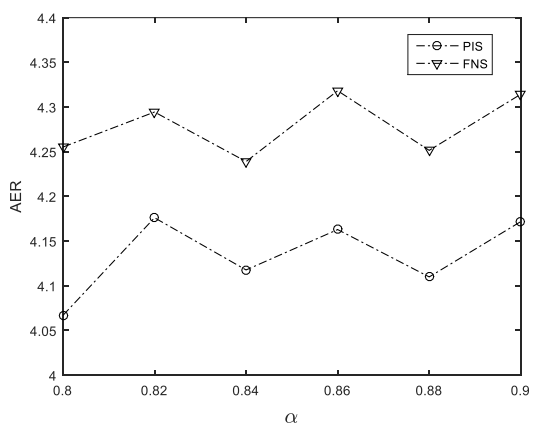

(d) AER

Fig. 6. Comparison results of Simulation methods I and II in parameters scenario (iii)

\section{Conclusion}

This paper investigates the PIS-based FMEA problem, where FMEA members express LDAMs over failure modes and risk factors and incomplete APRs over failure modes. We develop the three-step resolution procedure to deal with the PIS-based linguistic FMEA problem. Specifically, we present a two-stage optimization model to preprocess incomplete APRs to improve the reliability of the used preference data. Then, we propose a deviation minimum based optimization model to personalize individual semantics of FMEA members in their provided LDAMs. Finally, we present a ranking process to obtain the risk ordering of failure modes.

We validated the feasibility and validity of the PIS-based linguistic FMEA approach through a case study and detailed comparison analysis. Compared with the FNS-based linguistic FMEA approach, our model presents a novel linguistic FMEA approach to manage PIS, which is very helpful for risk analysts to recognize the most serious failure modes for enhancing system reliability and safety in linguistic decision contexts.

In future, the following issues need attention:

(1) In practice, the opinions of FMEA members may differ from each other to a large extent, which will weaken the reliability-management quality [44]. We argue that it will be interesting to integrate a consensus reaching process into the PIS-based linguistic FMEA approach to assist FMEA members to achieve a consensus on the final risk ordering of failure modes [33].

(2) Psychological behaviors, such as non-cooperative behaviors [35] and prospect theory [41], of FMEA members play an important role in practical FMEA problems. So, it would be interesting 
to incorporate psychological behaviors of FMEA participants in the PIS-based linguistic FMEA approach.

(3) The increasing complexity of reliability management results in the FMEA problem with a large number of members involved (large-scale FMEA problem) [25]. So, it would be interesting to investigate the PIS-based linguistic FMEA problem in a large-scale context based on the latest research results from a large-scale GDM problem $[17,26]$.

\section{References}

[1] J.R. Bradley, H.H. Guerrero, An alternative FMEA method for simple and accurate ranking of failure modes, Decision Sciences 42 (2011) 743-771.

[2] M. Braglia, M. Frosolini, R. Montanari, Fuzzy TOPSIS approach for failure mode, effects and criticality analysis, Quality and Reliability Engineering International 19 (2003) 425-443.

[3] M.C. Cantone, M. Ciocca, F. Dionisi, P. Fossati, S. Lorentini, M. Krengli, S. Molinelli, R. Orecchia, M. Schwarz, I. Veronese, Application of failure mode and effects analysis to treatment planning in scanned proton beam radiotherapy, Radiation Oncology 8 (2013) 127.

[4] S. Carpitella, A. Certa, J. Izquierdo, C.M. La Fata, A combined multi-criteria approach to support FMECA analyses: A real-world case, Reliability Engineering \& System Safety 169 (2018) 394-402.

[5] A. Certa, M. Enea, G.M. Galante, C.M. La Fata, ELECTRE TRI-based approach to the failure modes classification on the basis of risk parameters: An alternative to the risk priority number, Computers \& Industrial Engineering 108 (2017) 100-110.

[6] S.X. Chen, J.Q. Wang, T.L. Wang, Cloud-based ERP system selection based on extended probabilistic linguistic MULTIMOORA method and Choquet integral operator, Computational and Applied Mathematics 38 (2019) 88.

[7] Y.C. Dong, G.Q. Zhang, W.C. Hong, S. Yu, Linguistic computational model based on 2-tuples and intervals, IEEE Transactions on Fuzzy Systems 21 (2013) 1006-1018.

[8] M. Fedrizzi, S. Giove, Incomplete pairwise comparison and consistency optimization, European Journal of Operational Research 183 (2007) 303-313.

[9] M. Forghani-Elahabad, N. Kagan, Reliability evaluation of a stochastic-flow network in terms of minimal paths with budget constraint, IISE Transactions 51 (2019) 547-558.

[10] F. Franceschini, M. Galetto, A new approach for evaluation of risk priorities of failure modes in FMEA, International Journal of Production Research 39 (2001) 2991-3002.

[11] H. Gargama, S.K. Chaturvedi, Criticality assessment models for failure mode effects and criticality analysis using fuzzy logic, IEEE Transactions on Reliability 60 (2011) 102-110.

[12] H.H. Guerrero, J.R. Bradley, Failure modes and effects analysis: An evaluation of group versus individual performance, Production and Operations Management 22 (2013) 1524-1539.

[13] E. Herrera-Viedma, F. Chiclana, F. Herrera, S. Alonso, Group decision-making model with incomplete fuzzy preference relations based on additive consistency, IEEE Transactions on Systems, Man, and Cybernetics, Part B (Cybernetics) 37 (2007) 176-189.

[14] J. Huang, Z.J. Li, H.C. Liu, New approach for failure mode and effect analysis using linguistic distribution assessments and TODIM method, Reliability Engineering \& System Safety 167 (2017) 302-309.

[15] R.R. Inman, D.E. Blumenfeld, N. Huang, J. Li, J. Li, Survey of recent advances on the interface 
between production system design and quality, IIE Transactions 45 (2013) 557-574.

[16] P. Ji, H. Zhang, J. Wang, A fuzzy decision support model with sentiment analysis for items comparison in e-commerce: The case study of PConline.com, IEEE Transactions on Systems, Man, and Cybernetics: Systems, in press (2018) doi: 10.1109/TSMC.2018.2875163.

[17] C.C. Li, Y.C. Dong, F. Herrera, A consensus model for large-scale linguistic group decision making with a feedback recommendation based on clustered personalized individual semantics and opposing consensus groups, IEEE Transactions on Fuzzy Systems 27 (2019) 221-233.

[18] C.C. Li, Y.C. Dong, F. Herrera, E. Herrera-Viedma, L. Martínez, Personalized individual semantics in computing with words for supporting linguistic group decision making. An application on consensus reaching, Information Fusion 33 (2017) 29-40.

[19] C.C. Li, R.M. Rodríguez, L. Martínez, Y.C. Dong, F. Herrera, Personalized individual semantics based on consistency in hesitant linguistic group decision making with comparative linguistic expressions, Knowledge-Based Systems 145 (2018) 156-165.

[20] J. Li, J.Q. Wang, Multi-criteria decision-making with probabilistic hesitant fuzzy information based on expected multiplicative consistency, Neural Computing and Applications, in press (2018) doi: 10.1007/s00521-00018-03753-00521.

[21] R.X. Liang, J.Q. Wang, A linguistic intuitionistic cloud decision support model with sentiment analysis for product selection in E-commerce, International Journal of Fuzzy Systems 21 (2019) 963-977.

[22] H.C. Liu, FMEA using uncertainty theories and MCDM methods, Springer, Singapore (2016).

[23] H.C. Liu, L. Liu, N. Liu, Risk evaluation approaches in failure mode and effects analysis: A literature review, Expert Systems with Applications 40 (2013) 828-838.

[24] H.C. Liu, J.X. You, S.M. Chen, Y.Z. Chen, An integrated failure mode and effect analysis approach for accurate risk assessment under uncertainty, IIE Transactions 48 (2016) 1027-1042.

[25] H.C. Liu, X.Y. You, F. Tsung, P. Ji, An improved approach for failure mode and effect analysis involving large group of experts: An application to the healthcare field, Quality Engineering 30 (2018) 762-775.

[26] Y. Liu, Z.P. Fan, X. Zhang, A method for large group decision-making based on evaluation information provided by participators from multiple groups, Information Fusion 29 (2016) 132-141.

[27] Y. Lu, F. Teng, J. Zhou, A.Q. Wen, Y.T. Bi, Failure mode and effect analysis in blood transfusion: a proactive tool to reduce risks, Transfusion 53 (2013) 3080-3087.

[28] S.Z. Luo, H.Y. Zhang, J.Q. Wang, L. Li, Group decision-making approach for evaluating the sustainability of constructed wetlands with probabilistic linguistic preference relations, Journal of the Operational Research Society, in press (2019) doi: 10.1080/01605682.01602018.01510806.

[29] K. Mccullough, N. Ebrahimi, Approximate Bayesian computation for censored data and its application to reliability assessment, IISE Transactions 50 (2018) 419-430.

[30] J.M. Mendel, D. Wu, Perceptual computing: Aiding people in making subjective judgments, Wiley and Sons, 2010.

[31] J.M. Mendel, L.A. Zadeh, E. Trillas, R. Yager, J. Lawry, H. Hagras, S. Guadarrama, What computing with words means to me, IEEE Computational Intelligence Magazine 5 (2010) 20-26.

[32] O. Mohsen, N. Fereshteh, An extended VIKOR method based on entropy measure for the failure modes risk assessment - A case study of the geothermal power plant (GPP), Safety Science 92 (2017) 160-172.

[33] E. Moreno-Centeno, A.R. Escobedo, Axiomatic aggregation of incomplete rankings, IIE 
Transactions 48 (2016) 475-488.

[34] R.X. Nie, Z.P. Tian, X.K. Wang, J.Q. Wang, T.L. Wang, Risk evaluation by FMEA of supercritical water gasification system using multi-granular linguistic distribution assessment, Knowledge-Based Systems 162 (2018) 185-201.

[35] I. Palomares, L. Martínez, F. Herrera, A consensus model to detect and manage noncooperative behaviors in large-scale group decision making, IEEE Transactions on Fuzzy Systems 22 (2014) 516-530.

[36] H. Peng, Q. Feng, D.W. Coit, Reliability and maintenance modeling for systems subject to multiple dependent competing failure processes, IIE Transactions 43 (2010) 12-22.

[37] R.K. Sharma, D. Kumar, P. Kumar, Systematic failure mode effect analysis (FMEA) using fuzzy linguistic modelling, International Journal of Quality \& Reliability Management 22 (2005) 986-1004.

[38] C. Spreafico, D. Russo, C. Rizzi, A state-of-the-art review of FMEA/FMECA including patents, Computer Science Review 25 (2017) 19-28.

[39] D.H. Stamatis, Failure mode and effect analysis: FMEA from theory to execution, ASQC Press, NewYork, NY (2003).

[40] W.Z. Wang, X.W. Liu, J.D. Qin, S.L. Liu, An extended generalized TODIM for risk evaluation and prioritization of failure modes considering risk indicators interaction, IISE Transactions 51 (2019) 1236-1250.

[41] W.Z. Wang, X.W. Liu, Y. Qin, Y. Fu, A risk evaluation and prioritization method for FMEA with prospect theory and Choquet integral, Safety Science 110 (2018) 152-163.

[42] X. Wang, J. Wang, H. Zhang, Distance-based multicriteria group decision-making approach with probabilistic linguistic term sets, Expert Systems 36 (2019) e12352.

[43] G.Q. Zhang, Y.C. Dong, Y.F. Xu, Consistency and consensus measures for linguistic preference relations based on distribution assessments, Information Fusion 17 (2014) 46-55.

[44] H.J. Zhang, Y.C. Dong, I. Palomares, H.W. Zhou, Failure mode and effect analysis in a linguistic context: A consensus-based multi-attribute group decision-making approach, IEEE Transactions on Reliability 68 (2019) 566-582.

[45] H.J. Zhang, J. Xiao, Y.C. Dong, Integrating a consensus-reaching mechanism with bounded confidences into failure mode and effect analysis under incomplete context, Knowledge-Based Systems 183 (2019) 104873.

[46] Q.J. Zhou, V.V. Thai, Fuzzy and grey theories in failure mode and effect analysis for tanker equipment failure prediction, Safety science 83 (2016) 74-79.

[47] Y.Q. Zhou, J.W. Xia, Y.T. Zhong, J.H. Pang, An improved FMEA method based on the linguistic weighted geometric operator and fuzzy priority, Quality Engineering 28 (2016) 491-498.

\section{Appendices}

Appendix A: Acronyms and Notations

Acronyms:

FMEA: Failure modes and effects analysis

PIS: Personalized individual semantics

LDAMs: Linguistic distribution assessment matrices 
NAMs: Numerical assessment matrices

APRs: Additive preference relations

RPN: Risk priority number

GDM: Group decision-making

FNS: Fixed numerical scale

PNS: PIS-based numerical scale

O: Occurrence

S: Severity

D: Detection

\section{Notations:}

$X=\left\{x_{1}, x_{2}, \ldots, x_{n}\right\}:$ Set of objects

$L=\left\{l_{0}, \ldots, l_{g}\right\}$ : Linguistic term set

$L A D=\left\{\left(l_{s}, \vartheta_{s}\right) \mid s=0,1, \ldots, g\right\}$ : Linguistic distribution assessment on set $L$

$N S\left(l_{i}\right)$ : Numerical scale of $l_{i}$

$F M=\left\{F M_{1}, F M_{2}, \ldots, F M_{n}\right\}:$ Set of identified failure modes

$R F=\left\{R F_{1}, R F_{2}, \ldots, R F_{y}\right\}:$ Set of risk factors

$\omega=\left(\omega_{1}, \omega_{2}, \ldots, \omega_{y}\right)^{T}:$ Weight vector of risk factors

$T M=\left\{T M_{1}, T M_{2}, \ldots, T M_{m}\right\}:$ Set of FMEA members

$\lambda=\left(\lambda_{1}, \lambda_{2}, \ldots, \lambda_{m}\right)^{T}:$ Weight vector of FMEA members

$U^{k}=\left(u_{i j}^{k}\right)_{n \times y}$ : Individual linguistic distribution assessment matrix provided by $T M_{k}$

$V^{k}=\left(v_{i j}^{k}\right)_{n \times y}$ : Individual numerical matrix associated with $U^{k}=\left(u_{i j}^{k}\right)_{n \times y}$

$\dot{A}^{k}=\left(\dot{a}_{i j}^{k}\right)_{n \times n}:$ Incomplete additive preference relation provided by $T M_{k}$

$A^{k}=\left(a_{i j}^{k}\right)_{n \times n}$ : Complete additive preference relation associated with $\dot{A}^{k}=\left(\dot{a}_{i j}^{k}\right)_{n \times n}$

$\alpha$ : Consistency threshold

$P N S^{k}\left(l_{i}\right)$ : PNS of the linguistic term $l_{i}$ associated with $T M_{k}$

$\varepsilon$ : Discriminant factor

$P V V^{k}$ : Individual preference vector associated with $V^{k}$

$P V A^{k}$ : Individual preference vector associated with $A^{k}$

$R V^{k}$ : Individual risk ordering assicited with $P V V^{k}$

$R A^{k}$ : Individual risk ordering assicited with $P V A^{k}$

$P V V^{c}$ : Collective preference vector derived from $\left\{P V V^{1}, \ldots, P V V^{m}\right\}$

$P V A^{c}$ : Collective preference vector derived from $\left\{P V A^{1}, \ldots, P V A^{m}\right\}$

$E^{c}=\left(E_{1}^{c}, E_{2}^{c}, \ldots, E_{n}^{c}\right)^{T}$ : Overall preference vector obtained from $P V V^{c}$ and $P V A^{c}$

$R^{c}=\left(r_{1}^{c}, r_{2}^{c}, \ldots, r_{n}^{c}\right)^{T}$ : Collective risk ordering of failure modes associated with $E^{c}$ 


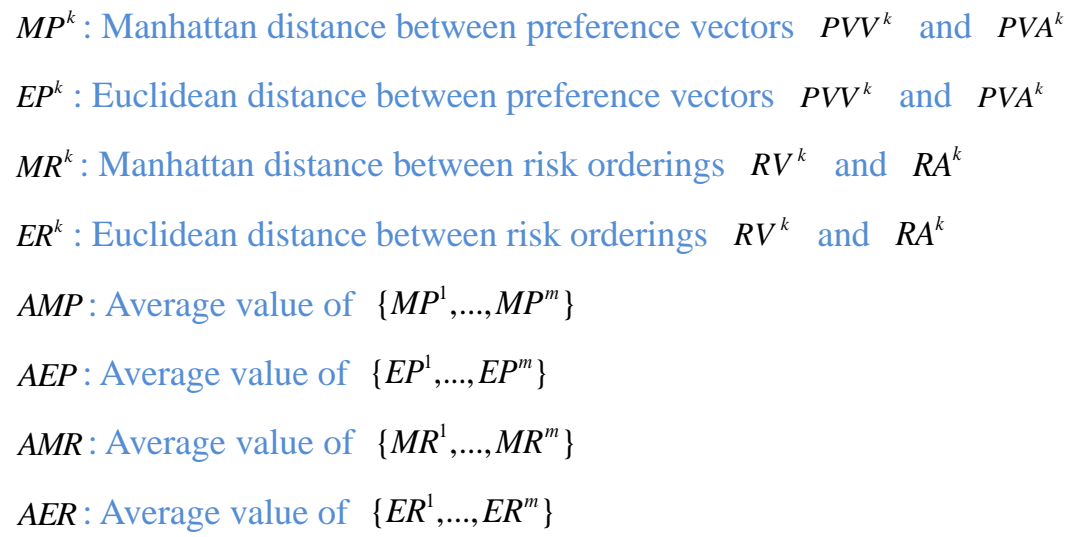

\section{Appendix B: Proofs}

\section{Proof of Proposition 1:}

In model $P_{2}$, constraints $(a)$ and $(b)$ guarantee that $\left|a_{i j}^{k}+a_{j z}^{k}-a_{i z}^{k}-0.5\right| \leq b_{i j z}^{k}$. The objective function guarantees that the optimal value is obtained when $\left|a_{i j}^{k}+a_{j z}^{k}-a_{i z}^{k}-0.5\right|=b_{i j z}^{k}$. So, model $P_{1}$ can be equivalently converted into the linear programming model $P_{2}$, which completes the proof.

\section{Proof of Proposition 2:}

Based on constraints (a) and (b), we have $\left|a_{i j}^{k}-a_{i j}^{k, *}\right| \leq b_{i j}^{k}$ in model $P_{4}$, and thus the optimal value of the objective function is produced only when $\left|a_{i j}^{k}-a_{i j}^{k, *}\right|=b_{i j}^{k}$. Moreover, according to constraints (c) and (d), we have $\left|a_{i j}^{k}+a_{j z}^{k}-a_{i z}^{k}-0.5\right| \leq c_{i j z}^{k}$. From constraint (e), we have $1-\frac{4}{n(n-1)(n-2)} \sum_{i, j, z=1 ; i<j<z}^{n}\left|a_{i j}^{k}+a_{j z}^{k}-a_{i z}^{k}-0.5\right| \geq 1-\frac{4}{n(n-1)(n-2)} \sum_{i, j, z=1 ; i<j<z}^{n} c_{i j z}^{k} \geq \alpha$. Therefore, model $P_{3}$ can be equivalently transformed into the linear programming model $P_{4}$. This completes the proof.

\section{Proof of Proposition 3:}

(i) Let $\Omega$ be the feasible region of model $P_{3}$. Then, we have $k d\left(A^{k}, A^{k, *}\right)=\frac{1}{\left\|K P^{k}\right\|} \sum_{(i, j) \in K P^{k}}\left|a_{i j}^{k}-a_{i j}^{k, *}\right| \geq 0$ for any $A^{k} \in \Omega$. Since $A^{k, *}$ satisfies the constraints of model $P_{3}$, then $A^{k, *} \in \Omega$. Moreover, $k d\left(A^{k, *}, A^{k, *}\right)=\frac{1}{\left\|K P^{k}\right\|} \sum_{(i, j) \in K P^{k}}\left|a_{i j}^{k, *^{*}}-a_{i j}^{k, *}\right|=0$, which means that the optimal objective function value of model $P_{3}$ is 0 . So, $A^{k, * * *}=\left(a_{i j}^{k, * * *}\right)_{n \times n}$ satisfies $a_{i j}^{k, * * *}=a_{i j}^{k, *}$ for $(i, j) \in K P^{k}$. Further, we have $k d\left(A^{k,,^{* *}}, A^{k, *}\right)=\frac{1}{\left\|K P^{k}\right\|_{(i, j) \in K P^{k}}}\left|a_{i j}^{k, * * *}-a_{i j}^{k, *}\right|=0$.

Let us assume that $k d\left(A^{k, * *}, A^{k,{ }^{*}}\right)=0$ when $C L\left(A^{k, *^{*}}\right)<\alpha$. Clearly, it is $C L\left(A^{k, * *}\right) \geq \alpha$ 
because $A^{k, * *}$ is the optimal solution of model $P_{3}$. From $k d\left(A^{k, * *}, A^{k, *}\right)=0$ we have that $a_{i j}^{k, * *}=a_{i j}^{k, *}$ for $(i, j) \in K P^{k}$. So, $A^{k, * *}$ is a feasible solution of model $P_{1}$ because $A^{k, * *}$ satisfies the constraints of model $P_{1}$. Further, we can obtain that $C L\left(A^{k, *}\right) \geq C L\left(A^{k, * *}\right) \geq \alpha$, which violates the assumption that $k d\left(A^{k, *^{* *}}, A^{k, *}\right)=0$ when $C L\left(A^{k,{ }^{*}}\right)<\alpha$. Based on the above analysis, we have that $k d\left(A^{k, *^{* *}}, A^{k, *}\right)>0$ when $C L\left(A^{k, *}\right)<\alpha$.

This completes the proof of (i).

(ii) Recall that $A^{k, *}=\left(a_{i j}^{k, *}\right)_{n \times n}$ is the optimal complete APR associated with $\dot{A}^{k}=\left(\dot{a}_{i j}^{k}\right)_{n \times n}$ derived from model $P_{1}$. The following three possible cases are considered:

Case A: $C L\left(A^{k, *}\right) \geq \alpha_{1}>\alpha_{2}$. In this case, according to Proposition 3 (i) it is $d^{1,{ }^{*}}=0$ and $d^{2, *}=0$

Case B: $\alpha_{1}>C L\left(A^{k,{ }^{*}}\right) \geq \alpha_{2}$. In this case, according to Proposition 3 (i) it is $d^{1,{ }^{*}}>0$ and $d^{2, *}=0$

Case C: $\alpha_{1}>\alpha_{2}>C L\left(A^{k, *}\right)$. Let $\Omega_{1}$ and $\Omega_{2}$ be the feasible regions of model $P_{3}$ with $\alpha_{1}$ and $\alpha_{2}$, respectively. It is clear that $C L\left(A^{k}\right) \geq \alpha_{1}$ for any $A^{k} \in \Omega_{1}$. Because $\alpha_{1}>\alpha_{2}$, then $C L\left(A^{k}\right) \geq \alpha_{2}$ for any $A^{k} \in \Omega_{1}$, which means that $\Omega_{1} \subset \Omega_{2}$. Further, we heve $d^{1, *}>d^{2, *}$.

According to Cases A, B and C, we have that $d^{1, *} \geq d^{2, *}$ if $\alpha_{1}>\alpha_{2}$.

This completes the proof of (ii).

\section{Proof of Proposition 4:}

Since $\quad \sum_{i=1}^{n}\left|B_{i}^{k}-P V A_{i}^{k} \sum_{j=1}^{n} B_{j}^{k}\right|=0 \quad$ and $\quad\left|B_{i}^{k}-P V A_{i}^{k} \sum_{j=1}^{n} B_{j}^{k}\right| \geq 0 \quad$, we have that $\left|B_{i}^{k}-P V A_{i}^{k} \sum_{j=1}^{n} B_{j}^{k}\right|=0$ for $i=1,2, \ldots, n$. Further, we have that $P V A_{i}^{k}=B_{i}^{k} / \sum_{j=1}^{n} B_{j}^{k}=P V V_{i}^{k}$ $(i=1,2, \ldots, n)$. So, $P V V^{k}=P V A^{k}$, and $P V V^{k}$ and $P V A^{k}$ will yield the same risk orderings, i.e., $R V^{k}=R A^{k}$. This completes the proof of Proposition 4.

\section{Appendix C: Data and materials of case study (Section 5)}

\section{(1) The four failure modes}

Table A1: Four failure modes and their failure causes and effects in the blood transfusion process

\begin{tabular}{|c|c|c|c|}
\hline No. & Failure mode & Failure cause & Failure effect \\
\hline$F M_{1}$ & $\begin{array}{l}\text { Insufficient and/ } \\
\text { or incorrect } \\
\text { clinical } \\
\text { information on } \\
\text { the request form }\end{array}$ & $\begin{array}{l}\text { Request form filled out } \\
\text { incorrectly/incompletely; patient } \\
\text { provided incorrect blood group }\end{array}$ & $\begin{array}{l}\text { The normal process is } \\
\text { interrupted; transfusion } \\
\text { cannot be performed within } \\
\text { an appropriate time frame }\end{array}$ \\
\hline$F M_{2}$ & $\begin{array}{l}\text { Insufficient } \\
\text { preoperative } \\
\text { assessment of the } \\
\text { blood product }\end{array}$ & $\begin{array}{l}\text { Improper evaluation of the disease or } \\
\text { potential blood loss }\end{array}$ & $\begin{array}{l}\text { Adverse event if } \\
\text { compatible blood cannot be } \\
\text { prepared in time after } \\
\text { emergency cross-matching }\end{array}$ \\
\hline
\end{tabular}


requirement

$F_{3} \quad \begin{aligned} & \text { Blood group } \\ & \text { verification }\end{aligned}$ incomplete

Transfusion

$F_{4}$ cannot be the appropriate time procedure

Importance of performing blood group testing on two separate occasions not recognized; use of another sample collected separately or historical records

Transfusion not started when blood products are sent to the clinic area; inappropriate transfusion time
ABO-incompatible transfusion reaction if no historical blood type or another sample for verification

Transfusion is delayed and patients receive uncertain quality blood products

(2) The seven-grade linguistic terms set $L$

Table A2: Seven-grade linguistic term set, $L$, used by FMEA members

\begin{tabular}{llllll}
\hline & $\boldsymbol{O}$ & & $\boldsymbol{S}$ & \\
\cline { 5 - 5 }$l_{0}$ & Very low & & Very slight & & Very unlikely \\
$l_{1}$ & Low & & Slight & & Unlikely \\
$l_{2}$ & Reasonably low & & Reasonably & & Reasonably \\
& & & slight & unlikely \\
$l_{3}$ & Average & Moderate & & Average \\
$l_{4}$ & Reasonably & Reasonably high & Reasonably likely \\
& frequent & & High & Likely \\
$l_{5}$ & Frequent & Very high & Very likely \\
$l_{6}$ & Very frequent & & & \\
\hline
\end{tabular}

(3) LDAMs $\left\{U^{1}, U^{2}, \ldots, U^{16}\right\}$

Table A3: LDAMs $\left\{U^{1}, U^{2}, \ldots, U^{16}\right\}$

\begin{tabular}{|c|c|c|c|c|c|c|c|}
\hline & \multicolumn{3}{|c|}{$U^{1}$} & \multicolumn{4}{|c|}{$U^{2}$} \\
\hline \multirow[b]{2}{*}{$F M_{1}$} & $\boldsymbol{O}$ & $S$ & $D$ & $\boldsymbol{O}$ & $S$ & & $D$ \\
\hline & $\begin{array}{l}\left\{\left(l_{2}, 0.1\right),\left(l_{3}, 0.2\right),\right. \\
\left.\left(l_{4}, 0.4\right),\left(l_{5}, 0.3\right)\right\}\end{array}$ & $\begin{array}{l}\left\{\left(l_{2}, 0.2\right),\left(l_{3}, 0.2\right)\right. \\
\left.\left(l_{4}, 0.4\right),\left(l_{5}, 0.2\right)\right\}\end{array}$ & $\begin{array}{l}\left\{\left(l_{1}, 0.1\right),\left(l_{2}, 0.2\right),\right. \\
\left(l_{3}, 0.2\right),\left(l_{4}, 0.2\right), \\
\left.\left(l_{5}, 0.3\right)\right\}\end{array}$ & $\begin{array}{l}\left\{\left(l_{2}, 0.15\right),\left(l_{3}, 0.2\right),\right. \\
\left.\left(l_{4}, 0.4\right),\left(l_{5}, 0.25\right)\right\}\end{array}$ & $\begin{array}{l}\left\{\left(l_{2}, 0.25\right),\left(l_{3}, 0.2\right),\right. \\
\left.\left(l_{4}, 0.4\right),\left(l_{5}, 0.15\right)\right\}\end{array}$ & \multicolumn{2}{|c|}{$\begin{array}{l}\left\{\left(l_{1}, 0.15\right),\left(l_{2}, 0.2\right),\right. \\
\left(l_{3}, 0.2\right),\left(l_{4}, 0.2\right), \\
\left.\left(l_{5}, 0.25\right)\right\}\end{array}$} \\
\hline$F M_{2}$ & $\begin{array}{l}\left\{\left(l_{3}, 0.1\right),\left(l_{4}, 0.4\right),\right. \\
\left.\left(l_{5}, 0.2\right),\left(l_{6}, 0.3\right)\right\}\end{array}$ & $\begin{array}{l}\left\{\left(l_{1}, 0.1\right),\left(l_{2}, 0.2\right),\right. \\
\left(l_{3}, 0.1\right),\left(l_{4}, 0.3\right), \\
\left.\left(l_{5}, 0.2\right),\left(l_{6}, 0.1\right)\right\}\end{array}$ & $\begin{array}{l}\left\{\left(l_{3}, 0.3\right),\left(l_{4}, 0.2\right)\right. \\
\left.\left(l_{5}, 0.3\right),\left(l_{6}, 0.2\right)\right\}\end{array}$ & $\begin{array}{l}\left\{\left(l_{3}, 0.15\right),\left(l_{4}, 0.4\right),\right. \\
\left.\left(l_{5}, 0.2\right),\left(l_{6}, 0.25\right)\right\}\end{array}$ & $\begin{array}{l}\left\{\left(l_{1}, 0.15\right),\left(l_{2}, 0.2\right),\right. \\
\left(l_{3}, 0.1\right),\left(l_{4}, 0.3\right), \\
\left.\left(l_{5}, 0.2\right),\left(l_{6}, 0.05\right)\right\}\end{array}$ & \multicolumn{2}{|c|}{$\begin{array}{l}\left\{\left(l_{3}, 0.35\right),\left(l_{4}, 0.2\right),\right. \\
\left.\left(l_{5}, 0.3\right),\left(l_{6}, 0.15\right)\right\}\end{array}$} \\
\hline$F M_{3}$ & $\begin{array}{l}\left\{\left(l_{1}, 0.2\right),\left(l_{2}, 0.2\right),\right. \\
\left.\left(l_{3}, 0.6\right)\right\}\end{array}$ & $\begin{array}{l}\left\{\left(l_{2}, 0.2\right),\left(l_{3}, 0.4\right),\right. \\
\left.\left(l_{4}, 0.4\right)\right\}\end{array}$ & $\begin{array}{l}\left\{\left(l_{1}, 0.1\right),\left(l_{2}, 0.4\right),\right. \\
\left.\left(l_{3}, 0.3\right),\left(l_{4}, 0.2\right)\right\}\end{array}$ & $\begin{array}{l}\left\{\left(l_{1}, 0.25\right),\left(l_{2}, 0.2\right)\right. \\
\left.\left(l_{3}, 0.55\right)\right\}\end{array}$ & $\begin{array}{l}\left\{\left(l_{2}, 0.25\right),\left(l_{3}, 0.4\right),\right. \\
\left.\left(l_{4}, 0.35\right)\right\}\end{array}$ & \multicolumn{2}{|c|}{$\begin{array}{l}\left\{\left(l_{1}, 0.15\right),\left(l_{2}, 0.4\right),\right. \\
\left.\left(l_{3}, 0.3\right),\left(l_{4}, 0.15\right)\right\}\end{array}$} \\
\hline \multirow[t]{3}{*}{$\mathrm{FM}_{4}$} & $\begin{array}{l}\left\{\left(l_{0}, 0.1\right),\left(l_{1}, 0.4\right),\right. \\
\left.\left(l_{2}, 0.3\right),\left(l_{3}, 0.2\right)\right\}\end{array}$ & $\begin{array}{l}\left\{\left(l_{1}, 0.1\right),\left(l_{2}, 0.5\right),\right. \\
\left.\left(l_{3}, 0.1\right),\left(l_{4}, 0.3\right)\right\}\end{array}$ & $\begin{array}{l}\left\{\left(l_{1}, 0.2\right),\left(l_{2}, 0.2\right),\right. \\
\left.\left(l_{3}, 0.2\right),\left(l_{4}, 0.4\right)\right\}\end{array}$ & $\begin{array}{l}\left\{\left(l_{0}, 0.15\right),\left(l_{1}, 0.4\right),\right. \\
\left.\left(l_{2}, 0.3\right),\left(l_{3}, 0.15\right)\right\}\end{array}$ & $\begin{array}{l}\left\{\left(l_{1}, 0.15\right),\left(l_{2}, 0.5\right),\right. \\
\left.\left(l_{3}, 0.1\right),\left(l_{4}, 0.25\right)\right\}\end{array}$ & \multicolumn{2}{|c|}{$\begin{array}{l}\left\{\left(l_{1}, 0.25\right),\left(l_{2}, 0.2\right)\right. \\
\left.\left(l_{3}, 0.2\right),\left(l_{4}, 0.35\right)\right\}\end{array}$} \\
\hline & \multicolumn{3}{|c|}{$U^{3}$} & \multicolumn{4}{|c|}{$U^{4}$} \\
\hline & $\boldsymbol{O}$ & $S$ & $D$ & $O$ & $S$ & & $D$ \\
\hline$F M_{1}$ & $\begin{array}{l}\left\{\left(l_{1}, 0.25\right),\left(l_{2}, 0.2\right),\right. \\
\left.\left(l_{3}, 0.3\right),\left(l_{4}, 0.25\right)\right\}\end{array}$ & $\begin{array}{l}\left\{\left(l_{1}, 0.15\right),\left(l_{2}, 0.3\right),\right. \\
\left.\left(l_{3}, 0.2\right),\left(l_{4}, 0.35\right)\right\}\end{array}$ & $\begin{array}{l}\left\{\left(l_{1}, 0.2\right),\left(l_{2}, 0.45\right),\right. \\
\left.\left(l_{3}, 0.1\right),\left(l_{4}, 0.25\right)\right\}\end{array}$ & $\begin{array}{l}\left\{\left(l_{0}, 0.15\right),\left(l_{1}, 0.25\right),\right. \\
\left(l_{2}, 0.1\right),\left(l_{3}, 0.2\right), \\
\left.\left(l_{4}, 0.15\right),\left(l_{5}, 0.15\right)\right\}\end{array}$ & \multicolumn{2}{|c|}{$\begin{array}{l}\left\{\left(l_{0}, 0.15\right),\left(l_{1}, 0.35\right),\right. \\
\left(l_{2}, 0.05\right),\left(l_{3}, 0.2\right), \\
\left.\left(l_{4}, 0.2\right),\left(l_{5}, 0.05\right)\right\}\end{array}$} & $\begin{array}{l}\left\{\left(l_{0}, 0.1\right),\left(l_{1}, 0.35\right),\right. \\
\left(l_{2}, 0.25\right),\left(l_{3}, 0.05\right), \\
\left.\left(l_{4}, 0.2\right),\left(l_{5}, 0.05\right)\right\}\end{array}$ \\
\hline$F M_{2}$ & $\begin{array}{l}\left\{\left(l_{1}, 0.3\right),\left(l_{2}, 0.2\right),\right. \\
\left(l_{3}, 0.1\right),\left(l_{4}, 0.3\right), \\
\left.\left(l_{5}, 0.1\right)\right\}\end{array}$ & $\begin{array}{l}\left\{\left(l_{1}, 0.1\right),\left(l_{2}, 0.5\right),\right. \\
\left(l_{3}, 0.1\right),\left(l_{4}, 0.1\right), \\
\left.\left(l_{5}, 0.2\right)\right\}\end{array}$ & $\begin{array}{l}\left\{\left(l_{1}, 0.3\right),\left(l_{2}, 0.2\right),\right. \\
\left(l_{3}, 0.3\right),\left(l_{4}, 0.1\right) \\
\left.\left(l_{5}, 0.1\right)\right\}\end{array}$ & $\begin{array}{l}\left\{\left(l_{0}, 0.15\right),\left(l_{1}, 0.2\right),\right. \\
\left(l_{2}, 0.2\right),\left(l_{3}, 0.1\right), \\
\left.\left(l_{4}, 0.2\right),\left(l_{5}, 0.15\right)\right\}\end{array}$ & $\begin{array}{l}\left\{\left(l_{0}, 0.15\right),\left(l_{1}, \mathrm{C}\right.\right. \\
\left(l_{2}, 0.1\right),\left(l_{3}, 0.2\right. \\
\left(l_{4}, 0.25\right),\left(l_{5}, 0\right.\end{array}$ & & $\begin{array}{l}\left\{\left(l_{0}, 0.1\right),\left(l_{1}, 0.15\right),\right. \\
\left(l_{2}, 0.1\right),\left(l_{3}, 0.2\right), \\
\left.\left(l_{4}, 0.25\right),\left(l_{5}, 0.2\right)\right\}\end{array}$ \\
\hline
\end{tabular}


$\begin{array}{llll}F M_{3} & \left\{\left(l_{1}, 0.5\right),\left(l_{2}, 0.2\right),\right. & \left\{\left(l_{1}, 0.4\right),\left(l_{2}, 0.3\right),\right. & \left\{\left(l_{1}, 0.3\right),\left(l_{2}, 0.3\right),\right. \\ & \left.\left(l_{3}, 0.3\right)\right\} & \left.\left(l_{3}, 0.2\right),\left(l_{4}, 0.1\right)\right\} & \left.\left(l_{3}, 0.2\right),\left(l_{4}, 0.2\right)\right\}\end{array}$

$\left\{\left(l_{0}, 0.25\right),\left(l_{1}, 0.35\right), \quad\left\{\left(l_{0}, 0.3\right),\left(l_{1}, 0.25\right)\right.\right.$, $\left(l_{2}, 0.2\right),\left(l_{3}, 0.1\right), \quad\left(l_{2}, 0.3\right),\left(l_{3}, 0.05\right)$, $\left.\left(l_{4}, 0.05\right),\left(l_{5}, 0.05\right)\right\}$ $\left\{\left(l_{0}, 0.15\right),\left(l_{1}, 0.3\right)\right.$, $\left(l_{2}, 0.35\right),\left(l_{3}, 0.1\right)$ $\left.\left(l_{4}, 0.05\right),\left(l_{5}, 0.05\right)\right\}$

$F M_{4} \quad\left\{\left(l_{1}, 0.3\right),\left(l_{2}, 0.2\right), \quad\left\{\left(l_{2}, 0.3\right),\left(l_{3}, 0.25\right), \quad\left\{\left(l_{1}, 0.1\right),\left(l_{2}, 0.5\right)\right.\right.\right.$ $\left.\left(l_{3}, 0.3\right),\left(l_{4}, 0.2\right)\right\}$ $\left.\left(l_{4}, 0.45\right)\right\}$

$\left.\left(l_{3}, 0.2\right),\left(l_{4}, 0.2\right)\right\}$

$\left\{\left(l_{0}, 0.2\right),\left(l_{1}, 0.3\right)\right.$, $\left(l_{2}, 0.25\right),\left(l_{3}, 0.05\right)$,

$\left\{\left(l_{0}, 0.1\right),\left(l_{1}, 0.25\right)\right.$,

$\left\{\left(l_{0}, 0.2\right),\left(l_{1}, 0.25\right)\right.$ $\left.\left(l_{4}, 0.15\right),\left(l_{5}, 0.05\right)\right\}$

\begin{tabular}{llll}
\hline \multirow{3}{*}{$F M_{1}$} & \multicolumn{3}{c}{$U^{5}$} \\
\cline { 2 - 4 } & $\boldsymbol{O}$ & $\boldsymbol{S}$ & $\boldsymbol{D}$ \\
\cline { 2 - 4 } & $\left\{\left(l_{0}, 0.1\right),\left(l_{1}, 0.3\right)\right.$, & $\left\{\left(l_{0}, 0.1\right),\left(l_{1}, 0.3\right)\right.$, & $\left\{\left(l_{0}, 0.1\right),\left(l_{1}, 0.3\right)\right.$, \\
& $\left(l_{2}, 0.1\right),\left(l_{3}, 0.3\right)$, & $\left(l_{2}, 0.2\right),\left(l_{3}, 0.2\right)$, & $\left(l_{2}, 0.1\right),\left(l_{3}, 0.2\right)$, \\
& $\left.\left(l_{4}, 0.2\right)\right\}$ & $\left.\left(l_{4}, 0.2\right)\right\}$ & $\left.\left(l_{4}, 0.3\right)\right\}$
\end{tabular}

$F M_{2} \quad\left\{\left(l_{1}, 0.2\right),\left(l_{2}, 0.1\right)\right.$,

$\left\{\left(l_{1}, 0.15\right),\left(l_{2}, 0.1\right)\right.$,

$\left(l_{3}, 0.2\right),\left(l_{4}, 0.2\right)$, $\left(l_{3}, 0.2\right),\left(l_{4}, 0.4\right)$,

$\left\{\left(l_{1}, 0.1\right),\left(l_{2}, 0.1\right)\right.$,

$\left.\left(l_{5}, 0.2\right),\left(l_{6}, 0.1\right)\right\}$

$$
\left.\left(l_{5}, 0.15\right)\right\}
$$

$\left(l_{3}, 0.3\right),\left(l_{4}, 0.2\right)$

$\left.\left(l_{5}, 0.3\right)\right\}$

$F M_{3}\left\{\left(l_{0}, 0.15\right),\left(l_{1}, 0.05\right)\right.$,

$\left\{\left(l_{0}, 0.2\right),\left(l_{1}, 0.15\right)\right.$,

$\left(l_{2}, 0.1\right),\left(l_{3}, 0.3\right)$

$\left(l_{2}, 0.05\right),\left(l_{3}, 0.3\right)$,

$\left\{\left(l_{0}, 0.2\right),\left(l_{1}, 0.35\right)\right.$,

$\left.\left(l_{4}, 0.4\right)\right\}$

$\left.\left(l_{4}, 0.3\right)\right\}$

$\left(l_{2}, 0.1\right),\left(l_{3}, 0.15\right)$

$\left.\left(l_{4}, 0.2\right)\right\}$

$U$

$\left\{\left(l_{0}, 0.1\right),\left(l_{1}, 0.25\right)\right.$,

$\left(l_{2}, 0.1\right),\left(l_{3}, 0.2\right)$

$\left\{\left(l_{0}, 0.1\right),\left(l_{1}, 0.2\right)\right.$,

D

$\left.\left(l_{4}, 0.05\right),\left(l_{5}, 0.3\right)\right\}$

$\left(l_{2}, 0.1\right),\left(l_{3}, 0.2\right)$,

$\left\{\left(l_{0}, 0.1\right),\left(l_{1}, 0.2\right)\right.$,

$\left\{\left(l_{0}, 0.1\right),\left(l_{1}, 0.1\right)\right.$,

$\left.\left(l_{4}, 0.2\right),\left(l_{5}, 0.2\right)\right\}$

$\left(l_{2}, 0.1\right),\left(l_{3}, 0.1\right)$

$\left(l_{2}, 0.1\right),\left(l_{3}, 0.1\right)$,

$\left\{\left(l_{0}, 0.1\right),\left(l_{1}, 0.3\right)\right.$,

$\left.\left(l_{4}, 0.2\right),\left(l_{5}, 0.3\right)\right\}$

$\left.\left(l_{4}, 0.3\right),\left(l_{5}, 0.3\right)\right\}$

$\left\{\left(l_{0}, 0.2\right),\left(l_{1}, 0.3\right)\right.$,

$\left\{\left(l_{0}, 0.6\right),\left(l_{1}, 0.2\right)\right.$,

$\left.\left(l_{2}, 0.6\right)\right\}$

$\left.\left(l_{2}, 0.2\right),\left(l_{3}, 0.3\right)\right\}$

$\left.\left(l_{2}, 0.2\right)\right\}$

$\left\{\left(l_{0}, 0.3\right),\left(l_{3}, 0.2\right)\right.$,

$\left\{\left(l_{0}, 0.05\right),\left(l_{1}, 0.2\right)\right.$,

$\left.\left(l_{4}, 0.4\right),\left(l_{5}, 0.1\right)\right\}$

$\left(l_{2}, 0.1\right),\left(l_{3}, 0.2\right)$

$\left.\left(l_{4}, 0.2\right),\left(l_{5}, 0.25\right)\right\}$

$F M_{4} \quad\left\{\left(l_{1}, 0.3\right),\left(l_{2}, 0.45\right)\right.$,

$\left\{\left(l_{0}, 0.3\right),\left(l_{1}, 0.25\right), \quad\left\{\left(l_{0}, 0.25\right),\left(l_{1}, 0.25\right)\right.\right.$,

$\left\{\left(l_{0}, 0.1\right),\left(l_{1}, 0.3\right)\right.$,

$\left\{\left(l_{0}, 0.1\right),\left(l_{1}, 0.3\right)\right.$,

$\left\{\left(l_{0}, 0.2\right),\left(l_{1}, 0.2\right)\right.$

$\left.\left(l_{3}, 0.15\right),\left(l_{4}, 0.1\right)\right\}$

$\left(l_{2}, 0.2\right),\left(l_{3}, 0.1\right)$,

$\left(l_{2}, 0.25\right),\left(l_{3}, 0.2\right)$,

$\left(l_{2}, 0.2\right),\left(l_{3}, 0.15\right)$,

$\left(l_{2}, 0.3\right),\left(l_{3}, 0.1\right)$,

$\left(l_{2}, 0.2\right),\left(l_{3}, 0.1\right)$

$\left.\left(l_{4}, 0.15\right),\left(l_{5}, 0.1\right)\right\}$

$\left.\left(l_{4}, 0.2\right)\right\}$

$\left.\left(l_{4}, 0.1\right),\left(l_{5}, 0.2\right)\right\}$

\begin{tabular}{lll}
\multicolumn{3}{c}{$U^{8}$} \\
\hline $\boldsymbol{O}$ & $\boldsymbol{S}$ & $\boldsymbol{D}$ \\
\hline$\left\{\left(l_{2}, 0.1\right),\left(l_{3}, 0.3\right)\right.$, & $\left\{\left(l_{2}, 0.5\right),\left(l_{3}, 0.3\right)\right.$, & $\left\{\left(l_{1}, 0.2\right),\left(l_{2}, 0.5\right)\right.$, \\
$\left.\left(l_{4}, 0.6\right)\right\}$ & $\left.\left(l_{4}, 0.2\right)\right\}$ & $\left.\left(l_{3}, 0.1\right),\left(l_{4}, 0.2\right)\right\}$
\end{tabular}

$F M_{2} \quad\left\{\left(l_{3}, 0.1\right),\left(l_{4}, 0.3\right), \quad\left\{\left(l_{1}, 0.1\right),\left(l_{2}, 0.2\right), \quad\left\{\left(l_{4}, 0.2\right),\left(l_{5}, 0.6\right)\right.\right.\right.$,

$\left\{\left(l_{3}, 0.1\right),\left(l_{4}, 0.3\right)\right.$

$\left\{\left(l_{1}, 0.2\right),\left(l_{2}, 0.2\right)\right.$,

$\left\{\left(l_{5}, 0.4\right),\left(l_{6}, 0.6\right)\right\}$

$\left.\left(l_{5}, 0.6\right)\right\}$

$\left.\left(l_{3}, 0.3\right),\left(l_{4}, 0.4\right)\right\}$

$\left.\left(l_{6}, 0.2\right)\right\}$

$\left.\left(l_{5}, 0.6\right)\right\}$

$\left.\left(l_{3}, 0.4\right),\left(l_{4}, 0.2\right)\right\}$

$\left\{\left(l_{2}, 0.2\right),\left(l_{3}, 0.5\right)\right.$,

$\left\{\left(l_{4}, 0.4\right),\left(l_{5}, 0.5\right)\right.$,

$\left\{\left(l_{3}, 0.3\right),\left(l_{4}, 0.2\right)\right.$

$F M_{3} \quad\left\{\left(l_{1}, 0.1\right),\left(l_{2}, 0.1\right)\right.$

$\left\{\left(l_{4}, 0.4\right),\left(l_{5}, 0.6\right)\right\}$

$\left\{\left(l_{3}, 0.3\right),\left(l_{4}, 0.2\right)\right.$,

$\left.\left(l_{4}, 0.3\right)\right\}$

$\left.\left(l_{6}, 0.1\right)\right\}$

$\left.\left(l_{5}, 0.5\right)\right\}$

$F M_{4} \quad\left\{\left(l_{2}, 0.3\right),\left(l_{3}, 0.2\right), \quad\left\{\left(l_{4}, 0.2\right),\left(l_{5}, 0.8\right)\right\} \quad\left\{\left(l_{3}, 0.1\right),\left(l_{4}, 0.5\right)\right.\right.$,

$\left\{\left(l_{1}, 0.3\right),\left(l_{2}, 0.3\right)\right.$,

$\left\{\left(l_{1}, 0.4\right),\left(l_{2}, 0.1\right)\right.$

$\left\{\left(l_{1}, 0.2\right),\left(l_{2}, 0.1\right)\right.$,

$\left.\left(l_{4}, 0.5\right)\right\}$

$\left.\left(l_{5}, 0.3\right),\left(l_{6}, 0.1\right)\right\}$

$\left.\left(l_{3}, 0.2\right),\left(l_{4}, 0.2\right)\right\}$

$\left.\left(l_{3}, 0.1\right),\left(l_{4}, 0.4\right)\right\}$

$\left.\left(l_{3}, 0.2\right),\left(l_{4}, 0.5\right)\right\}$

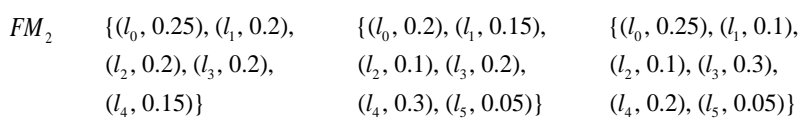

$F M_{3} \quad\left\{\left(l_{0}, 0.25\right),\left(l_{1}, 0.25\right), \quad\left\{\left(l_{0}, 0.15\right),\left(l_{1}, 0.35\right), \quad\left\{\left(l_{0}, 0.1\right),\left(l_{1}, 0.35\right)\right.\right.\right.$, $\left(l_{2}, 0.1\right),\left(l_{3}, 0.3\right), \quad\left(l_{2}, 0.05\right),\left(l_{3}, 0.3\right), \quad\left(l_{2}, 0.25\right),\left(l_{3}, 0.15\right)$, $\left.\left(l_{4}, 0.1\right)\right\}$ $\left.\left(l_{4}, 0.15\right)\right\}$

\begin{tabular}{lll} 
& $U^{10}$ & \\
\hline $\boldsymbol{O}$ & $\boldsymbol{S}$ & $\boldsymbol{D}$ \\
\hline$\left\{\left(l_{0}, 0.2\right),\left(l_{1}, 0.3\right)\right.$, & $\left\{\left(l_{0}, 0.2\right),\left(l_{1}, 0.3\right)\right.$, & $\left\{\left(l_{0}, 0.2\right),\left(l_{1}, 0.3\right)\right.$, \\
$\left(l_{2}, 0.1\right),\left(l_{3}, 0.3\right)$, & $\left(l_{2}, 0.2\right),\left(l_{3}, 0.2\right)$, & $\left(l_{2}, 0.1\right),\left(l_{3}, 0.2\right)$, \\
$\left.\left(l_{4}, 0.1\right)\right\}$ & $\left.\left(l_{4}, 0.1\right)\right\}$ & $\left.\left(l_{4}, 0.2\right)\right\}$
\end{tabular}

$\left\{\left(l_{0}, 0.2\right),\left(l_{1}, 0.2\right)\right.$, $\left(l_{2}, 0.25\right),\left(l_{3}, 0.2\right)$

$\left\{\left(l_{0}, 0.25\right),\left(l_{1}, 0.15\right), \quad\left\{\left(l_{0}, 0.3\right),\left(l_{1}, 0.1\right)\right.\right.$,

$\left.\left(l_{4}, 0.15\right)\right\}$ $\left(l_{2}, 0.1\right),\left(l_{3}, 0.2\right)$ $\left(l_{2}, 0.1\right),\left(l_{3}, 0.3\right)$,

$\left.\left(l_{4}, 0.3\right)\right\}$

$\left.\left(l_{4}, 0.2\right)\right\}$

$\left\{\left(l_{0}, 0.2\right),\left(l_{1}, 0.05\right), \quad\left\{\left(l_{0}, 0.25\right),\left(l_{1}, 0.15\right), \quad\left\{\left(l_{0}, 0.15\right),\left(l_{1}, 0.35\right)\right.\right.\right.$, $\left(l_{2}, 0.1\right),\left(l_{3}, 0.3\right), \quad\left(l_{2}, 0.05\right),\left(l_{3}, 0.3\right), \quad\left(l_{2}, 0.15\right),\left(l_{3}, 0.15\right)$, $\left.\left.\left.\left(l_{4}, 0.3\right),\left(l_{5}, 0.05\right)\right\} \quad\left(l_{4}, 0.2\right),\left(l_{5}, 0.05\right)\right\} \quad\left(l_{4}, 0.1\right),\left(l_{5}, 0.1\right)\right\}$ 
$\begin{array}{ll}F M_{4} \quad\left\{\left(l_{0}, 0.1\right),\left(l_{1}, 0.3\right),\right. \\ & \left(l_{2}, 0.25\right),\left(l_{3}, 0.15\right),\end{array}$ $\left.\left(l_{4}, 0.2\right)\right\}$ $\left\{\left(l_{0}, 0.25\right),\left(l_{1}, 0.25\right), \quad\left\{\left(l_{0}, 0.2\right),\left(l_{1}, 0.25\right)\right.\right.$, $\left(l_{2}, 0.2\right),\left(l_{3}, 0.1\right), \quad\left(l_{2}, 0.25\right),\left(l_{3}, 0.2\right)$, $\left.\left(l_{4}, 0.2\right)\right\}$ $\left.\left(l_{4}, 0.1\right)\right\}$ $\left\{\left(l_{0}, 0.1\right),\left(l_{1}, 0.3\right)\right.$,

$\left\{\left(l_{0}, 0.4\right),\left(l_{1}, 0.25\right)\right.$,

$\left\{\left(l_{0}, 0.3\right),\left(l_{1}, 0.25\right)\right.$,

\section{$\left.\left(l_{2}, 0.45\right),\left(l_{3}, 0.15\right)\right\} \quad\left(l_{2}, 0.2\right),\left(l_{3}, 0.1\right)$,}

$\left.\left(l_{2}, 0.25\right),\left(l_{3}, 0.2\right)\right\}$

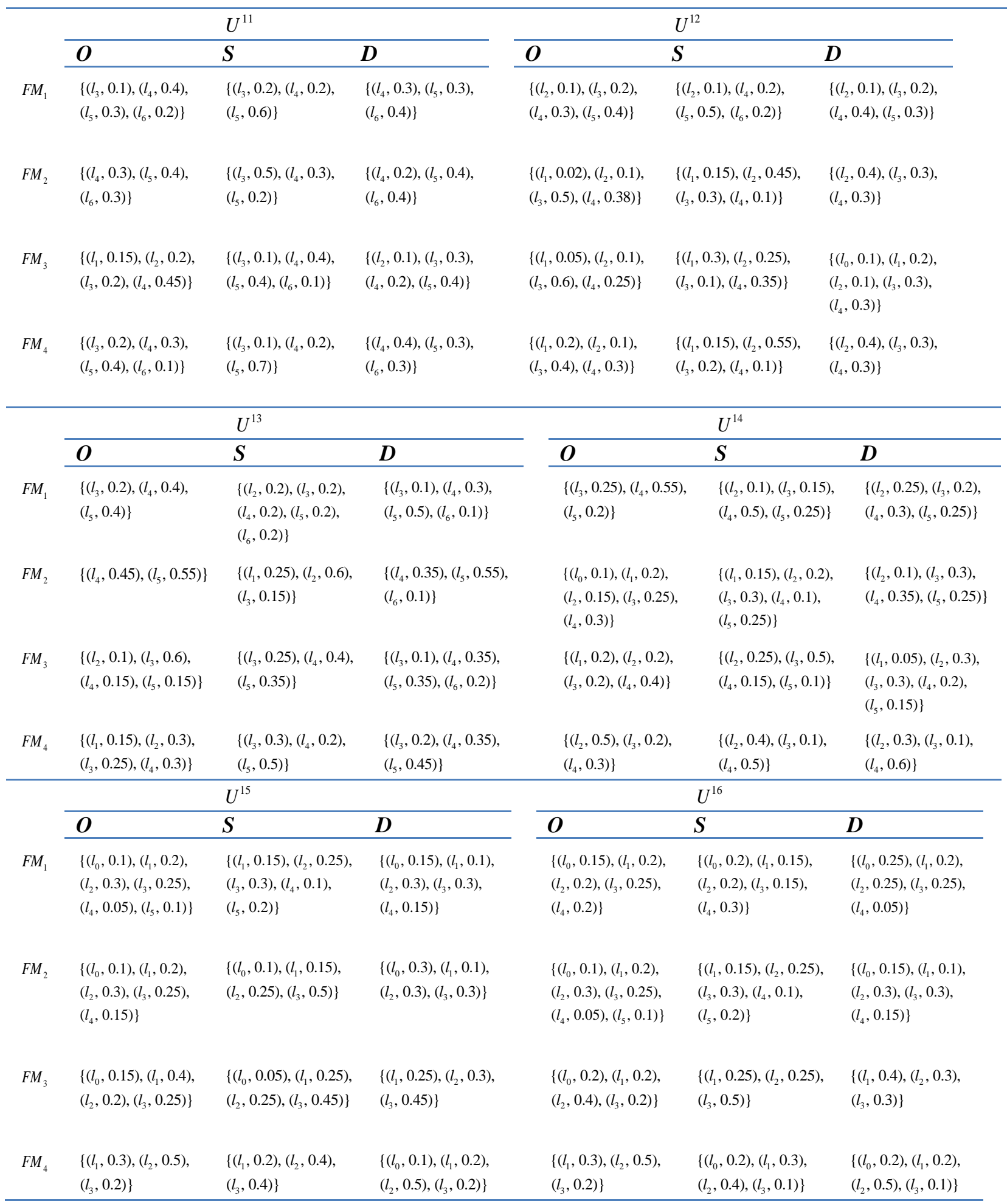

(4) Incomplete APRs $\left\{\dot{A}^{1}, \dot{A}^{2}, \ldots, \dot{A}^{16}\right\}$

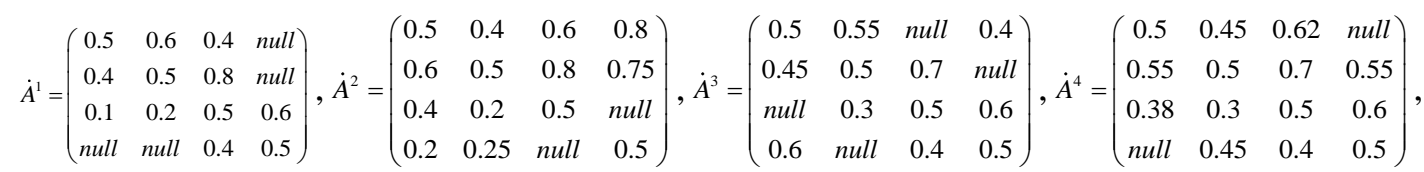


$\dot{A}^{5}=\left(\begin{array}{cccc}0.5 & 0.4 & \text { null } & 0.6 \\ 0.6 & 0.5 & 0.58 & 0.62 \\ \text { null } & 0.42 & 0.5 & \text { null } \\ 0.4 & 0.38 & \text { null } & 0.5\end{array}\right), \dot{A}^{6}=\left(\begin{array}{cccc}0.5 & \text { null } & 0.75 & 0.6 \\ \text { null } & 0.5 & 0.4 & \text { null } \\ 0.25 & 0.6 & 0.5 & 0.56 \\ 0.4 & \text { null } & 0.44 & 0.5\end{array}\right), \dot{A}^{7}=\left(\begin{array}{cccc}0.5 & \text { null } & 0.6 & 0.65 \\ \text { null } & 0.5 & 0.45 & \text { null } \\ 0.4 & 0.55 & 0.5 & 0.7 \\ 0.35 & \text { null } & 0.3 & 0.5\end{array}\right), \dot{A}^{8}=\left(\begin{array}{cccc}0.5 & \text { null } & 0.35 & 0.45 \\ \text { null } & 0.5 & 0.55 & \text { null } \\ 0.65 & 0.45 & 0.5 & 0.65 \\ 0.55 & \text { null } & 0.35 & 0.5\end{array}\right)$, $\dot{A}^{9}=\left(\begin{array}{cccc}0.5 & \text { null } & 0.7 & 0.5 \\ \text { null } & 0.5 & 0.46 & 0.62 \\ 0.3 & 0.54 & 0.5 & \text { null } \\ 0.5 & 0.38 & \text { null } & 0.5\end{array}\right), \dot{A}^{10}=\left(\begin{array}{cccc}0.5 & \text { null } & 0.42 & 0.62 \\ \text { null } & 0.5 & 0.46 & 0.55 \\ 0.58 & 0.54 & 0.5 & \text { null } \\ 0.38 & 0.45 & \text { null } & 0.5\end{array}\right), \dot{A}^{11}=\left(\begin{array}{cccc}0.5 & \text { null } & 0.65 & 0.58 \\ \text { null } & 0.5 & 0.72 & 0.58 \\ 0.35 & 0.28 & 0.5 & \text { null } \\ 0.42 & 0.42 & \text { null } & 0.5\end{array}\right), \dot{A}^{12}=\left(\begin{array}{cccc}0.5 & \text { null } & 0.58 & 0.76 \\ \text { null } & 0.5 & 0.48 & 0.55 \\ 0.42 & 0.52 & 0.5 & \text { null } \\ 0.24 & 0.45 & \text { null } & 0.5\end{array}\right)$, $\dot{A}^{13}=\left(\begin{array}{cccc}0.5 & 0.48 & 0.62 & \text { null } \\ 0.52 & 0.5 & \text { null } & 0.56 \\ 0.38 & \text { null } & 0.5 & 0.65 \\ \text { null } & 0.44 & 0.35 & 0.5\end{array}\right), \dot{A}^{14}=\left(\begin{array}{cccc}0.5 & 0.58 & 0.6 & \text { null } \\ 0.42 & 0.5 & \text { null } & 0.48 \\ 0.4 & \text { null } & 0.5 & 0.62 \\ \text { null } & 0.52 & 0.38 & 0.5\end{array}\right), \dot{A}^{15}=\left(\begin{array}{cccc}0.5 & 0.55 & 0.65 & \text { null } \\ 0.45 & 0.5 & \text { null } & 0.75 \\ 0.35 & \text { null } & 0.5 & 0.68 \\ \text { null } & 0.25 & 0.32 & 0.5\end{array}\right), \dot{A}^{16}=\left(\begin{array}{cccc}0.5 & 0.38 & 0.52 & \text { null } \\ 0.62 & 0.5 & \text { null } & 0.46 \\ 0.48 & \text { null } & 0.5 & 0.55 \\ \text { null } & 0.54 & 0.45 & 0.5\end{array}\right)$.

\section{(5) Complete APRs $\left\{A^{2}, A^{3}, \ldots, A^{16}\right\}$}

$A^{2}=\left(\begin{array}{cccc}0.5 & 0.4000 & 0.6000 & 0.8000 \\ 0.6000 & 0.5 & 0.8000 & 0.7500 \\ 0.4000 & 0.2000 & 0.5 & 0.5109 \\ 0.2000 & 0.2500 & 0.4891 & 0.5\end{array}\right), A^{3}=\left(\begin{array}{cccc}0.5 & 0.5151 & 0.5080 & 0.4323 \\ 0.4849 & 0.5 & 0.6547 & 0.5519 \\ 0.4920 & 0.3453 & 0.5 & 0.5626 \\ 0.5677 & 0.4481 & 0.4374 & 0.5\end{array}\right), A^{4}=\left(\begin{array}{cccc}0.5 & 0.4500 & 0.6200 & 0.5432 \\ 0.5500 & 0.5 & 0.7000 & 0.5500 \\ 0.3800 & 0.3000 & 0.5 & 0.6000 \\ 0.4568 & 0.4500 & 0.4000 & 0.5\end{array}\right)$, $A^{5}=\left(\begin{array}{cccc}0.5 & 0.4000 & 0.5007 & 0.6000 \\ 0.6000 & 0.5 & 0.5800 & 0.6200 \\ 0.4993 & 0.4200 & 0.5 & 0.5549 \\ 0.4000 & 0.3800 & 0.4451 & 0.5\end{array}\right), A^{6}=\left(\begin{array}{cccc}0.5 & 0.6945 & 0.7500 & 0.6000 \\ 0.3055 & 0.5 & 0.4000 & 0.4344 \\ 0.2500 & 0.6000 & 0.5 & 0.5600 \\ 0.400 & 0.5656 & 0.4400 & 0.5\end{array}\right), A^{7}=\left(\begin{array}{cccc}0.5 & 0.6105 & 0.6000 & 0.6500 \\ 0.3895 & 0.5 & 0.4500 & 0.6105 \\ 0.4000 & 0.5500 & 0.5 & 0.7000 \\ 0.3500 & 0.3895 & 0.3000 & 0.5\end{array}\right)$, $A^{8}=\left(\begin{array}{cccc}0.5 & 0.2847 & 0.3500 & 0.4500 \\ 0.7153 & 0.5 & 0.5500 & 0.6720 \\ 0.6500 & 0.4500 & 0.5 & 0.6500 \\ 0.5500 & 0.3280 & 0.3500 & 0.5\end{array}\right), A^{9}=\left(\begin{array}{cccc}0.5 & 0.5449 & 0.6835 & 0.5129 \\ 0.4551 & 0.5 & 0.4755 & 0.6049 \\ 0.3165 & 0.5245 & 0.5 & 0.4719 \\ 0.4871 & 0.3951 & 0.5281 & 0.5\end{array}\right), A^{10}=\left(\begin{array}{cccc}0.5 & 0.5057 & 0.4200 & 0.6200 \\ 0.4943 & 0.5 & 0.4600 & 0.5500 \\ 0.5800 & 0.5400 & 0.5 & 0.6067 \\ 0.3800 & 0.4500 & 0.3933 & 0.5\end{array}\right)$, $A^{11}=\left(\begin{array}{cccc}0.5 & 0.4803 & 0.6500 & 0.5800 \\ 0.5197 & 0.5 & 0.7200 & 0.5800 \\ 0.3500 & 0.2800 & 0.5 & 0.4185 \\ 0.4200 & 0.4200 & 0.5815 & 0.5\end{array}\right), A^{12}=\left(\begin{array}{cccc}0.5 & 0.6095 & 0.5800 & 0.7600 \\ 0.3905 & 0.5 & 0.4800 & 0.5500 \\ 0.4200 & 0.5200 & 0.5 & 0.5767 \\ 0.2400 & 0.4500 & 0.4233 & 0.5\end{array}\right), A^{13}=\left(\begin{array}{cccc}0.5 & 0.4800 & 0.6200 & 0.5682 \\ 0.5200 & 0.5 & 0.5033 & 0.5600 \\ 0.3800 & 0.4967 & 0.5 & 0.6500 \\ 0.4318 & 0.4400 & 0.3500 & 0.5\end{array}\right)$, $A^{14}=\left(\begin{array}{cccc}0.5 & 0.5800 & 0.6000 & 0.5817 \\ 0.4200 & 0.5 & 0.4796 & 0.4800 \\ 0.4000 & 0.5204 & 0.5 & 0.6200 \\ 0.4183 & 0.5200 & 0.3800 & 0.5\end{array}\right), A^{15}=\left(\begin{array}{cccc}0.5 & 0.5500 & 0.6500 & 0.8118 \\ 0.4500 & 0.5 & 0.5779 & 0.7500 \\ 0.3500 & 0.4221 & 0.5 & 0.6800 \\ 0.1882 & 0.2500 & 0.3200 & 0.5\end{array}\right), A^{16}=\left(\begin{array}{cccc}0.5 & 0.3800 & 0.5200 & 0.4992 \\ 0.6200 & 0.5 & 0.4994 & 0.4600 \\ 0.4800 & 0.5006 & 0.5 & 0.5500 \\ 0.5008 & 0.5400 & 0.4500 & 0.5\end{array}\right)$.

(6) NAMs $\left\{V^{1}, V^{2}, \ldots, V^{16}\right\}$ Table A4: NAMs $\left\{V^{1}, V^{2}, \ldots, V^{16}\right\}$

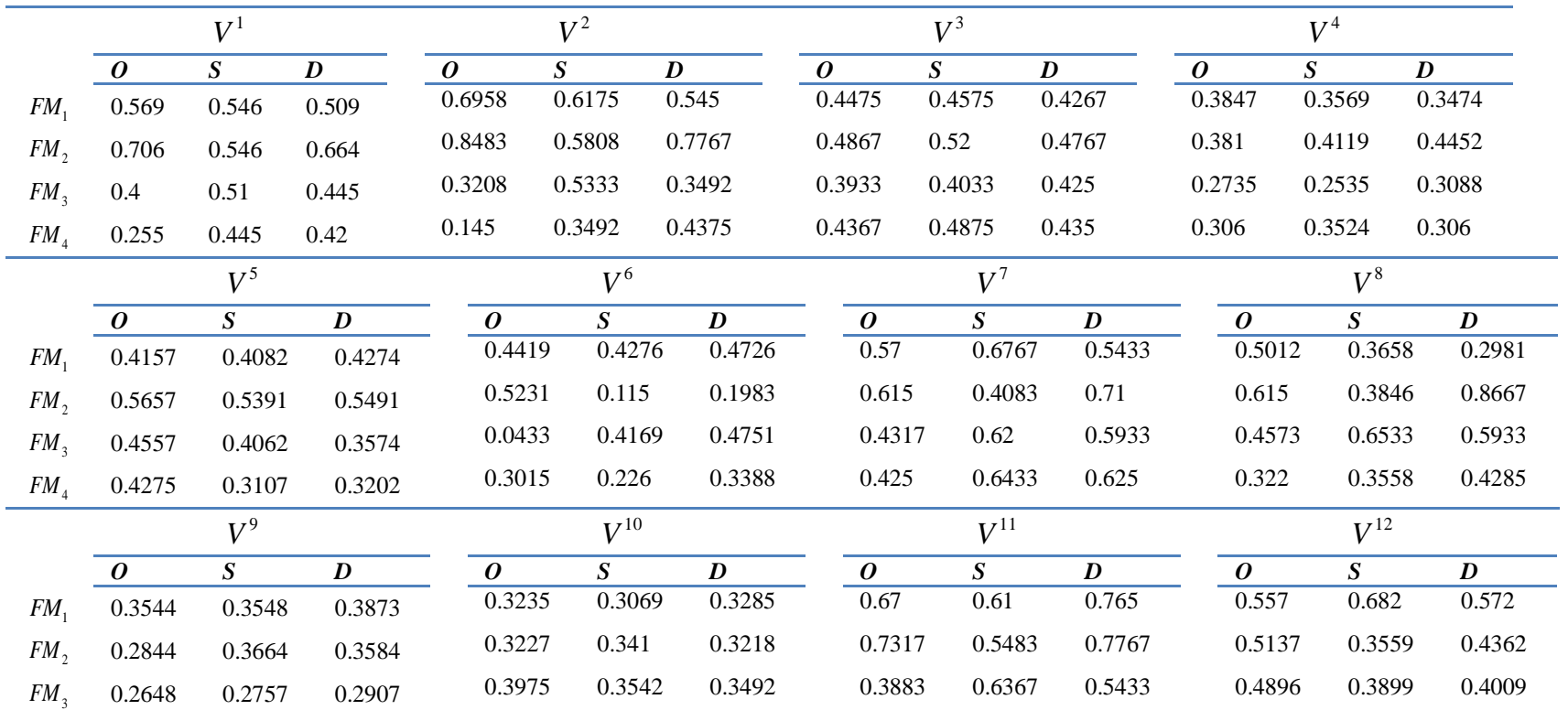




\begin{tabular}{|c|c|c|c|c|c|c|c|c|c|c|c|c|}
\hline$F M_{4}$ & 0.3153 & 0.2648 & 0.2823 & 0.3104 & 0.2152 & 0.2544 & 0.6317 & 0.6267 & 0.72 & 0.4509 & 0.3312 & 0.4362 \\
\hline & \multicolumn{3}{|c|}{$V^{13}$} & \multicolumn{3}{|c|}{$V^{14}$} & \multicolumn{3}{|c|}{$V^{15}$} & \multicolumn{3}{|c|}{$V^{16}$} \\
\hline & $O$ & $S$ & $D$ & $O$ & $S$ & $D$ & $O$ & $S$ & $D$ & $O$ & $S$ & $D$ \\
\hline$F M_{1}$ & 0.5867 & 0.6333 & 0.6483 & 0.5608 & 0.5371 & 0.4828 & 0.3215 & 0.4721 & 0.33 & 0.3919 & 0.3804 & 0.3317 \\
\hline$F M_{2}$ & 0.6142 & 0.4227 & 0.6592 & 0.3515 & 0.4357 & 0.5296 & 0.31 & 0.2992 & 0.205 & 0.4209 & 0.501 & 0.401 \\
\hline$F M_{3}$ & 0.5275 & 0.5783 & 0.6758 & 0.3918 & 0.4503 & 0.429 & 0.1783 & 0.2792 & 0.2875 & 0.3467 & 0.4458 & 0.4183 \\
\hline$F M_{4}$ & 0.4716 & 0.5933 & 0.5925 & 0.3672 & 0.4068 & 0.4413 & 0.1983 & 0.2767 & 0.1933 & 0.425 & 0.33 & 0.3417 \\
\hline
\end{tabular}

(7) Individual preference vectors obtained from $V^{k} \quad(k=1,2, \ldots, 16)$

$$
\begin{gathered}
P V V^{1}=(0.27,0.3185,0.2253,0.1862)^{T}, \quad P V V^{2}=(0.2998,0.3558,0.1941,0.1503)^{T}, \\
P V V^{3}=(0.2468,0.2749,0.2264,0.2519)^{T}, \quad P V V^{4}=(0.2638,0.3,0.2025,0.2337)^{T}, \\
P V V^{5}=(0.2414,0.3191,0.2353,0.2042)^{T}, \quad P V V^{6}=(0.3372,0.2101,0.235,0.2177)^{T}, \\
P V V^{7}=(0.2609,0.2526,0.2397,0.2468)^{T}, \quad P V V^{8}=(0.1994,0.3195,0.2917,0.1894)^{T}, \\
P V V^{9}=(0.2886,0.2656,0.2188,0.227)^{T}, \quad P V V^{10}=(0.2507,0.2576,0.2878,0.2039)^{T}, \\
P V V^{11}=(0.2674,0.2689,0.2051,0.2587)^{T}, \quad P V V^{12}=(0.3249,0.2317,0.2272,0.2162)^{T}, \\
P V V^{13}=(0.2668,0.2422,0.2544,0.2367)^{T}, \quad P V V^{14}=(0.2936,0.2446,0.2361,0.2257)^{T}, \\
P V V^{15}=(0.3353,0.243,0.2223,0.1994)^{T}, \quad P V V^{16}=(0.2332,0.2794,0.2557,0.2316)^{T} .
\end{gathered}
$$

(8) Individual preference vectors obtained from $A^{k} \quad(k=1,2, \ldots, 16)$

$$
\begin{gathered}
P V A^{1}=(0.27,0.3086,0.2401,0.1813)^{T}, \quad P V A^{2}=(0.3,0.3583,0.1851,0.1565)^{T}, \\
P V A^{3}=(0.2426,0.2819,0.2333,0.2422)^{T}, \quad P V A^{4}=(0.2689,0.3,0.2133,0.2178)^{T}, \\
P V A^{5}=(0.2501,0.3,0.2457,0.2042)^{T}, \quad P V A^{6}=(0.3408,0.19,0.235,0.2343)^{T}, \\
P V A^{7}=(0.3101,0.2417,0.275,0.1733)^{T}, \quad P V A^{8}=(0.1808,0.3229,0.2917,0.2047)^{T}, \\
P V A^{9}=(0.2902,0.2559,0.2188,0.2351)^{T}, \quad P V A^{10}=(0.2576,0.2507,0.2878,0.2039)^{T}, \\
P V A^{11}=(0.285,0.3033,0.1748,0.2369)^{T}, \quad P V A^{12}=(0.3249,0.2368,0.2528,0.1855)^{T}, \\
P V A^{13}=(0.278,0.2639,0.2544,0.2036)^{T}, \quad P V A^{14}=(0.2936,0.2299,0.2567,0.2197)^{T}, \\
P V A^{15}=(0.3353,0.2963,0.242,0.1264)^{T}, \quad P V A^{16}=(0.2332,0.2632,0.2551,0.2485)^{T} .
\end{gathered}
$$

Appendix D: Algorithm II and Simulation methods I and II

\section{(1) Algorithm II}

Algorithm II is obtained by replacing the Input and Step 2 of Algorithm I with the below Input' and Step 2', respectively.

Input': $\left\{U^{1}, U^{2}, \ldots, U^{m}\right\}, \quad\left\{\dot{A}^{1}, \dot{A}^{2}, \ldots, \dot{A}^{m}\right\}, \quad \lambda=\left(\lambda_{1}, \lambda_{2}, \ldots, \lambda_{m}\right)^{T}, \quad \omega=\left(\omega_{1}, \omega_{2}, \ldots, \omega_{y}\right)^{T}, \quad \alpha$, and $\tau$.

Step 2': Application of FNSs. Setting $N S^{k}\left(l_{t}\right)=t / g(k=1, \ldots, m ; t=0, \ldots, g)$. Then, transform $U^{k}=\left(u_{i j}^{k}\right)_{n \times y}$ into $V^{k}=\left(v_{i j}^{k}\right)_{n \times y}: v_{i j}^{k}=\sum_{t=0}^{g} \vartheta_{i j, t}^{k} \times N S^{k}\left(l_{t}\right)$. Next, let $P V V^{k}=\left(P V V_{1}^{k}, \ldots, P V V_{n}^{k}\right)^{T}$, where $\quad P V V_{i}^{k}=\sum_{j=1}^{y} \omega_{j} \times v_{i j}^{k} / \sum_{h=1}^{n} \sum_{j=1}^{y} \omega_{j} \times v_{h j}^{k} \quad$. Generate $P V A^{k}=\left(P V A_{1}^{k}, \ldots, P V A_{n}^{k}\right)^{T} \quad$ from $A^{k}=\left(a_{i j}^{k}\right)_{n \times n}: \quad P V A_{i}^{k}=\frac{2}{n^{2}-n} \sum_{j=1, j \neq i}^{n} a_{i j}^{k}$. 
(2) Simulation method I

Table A5: Simulation method I

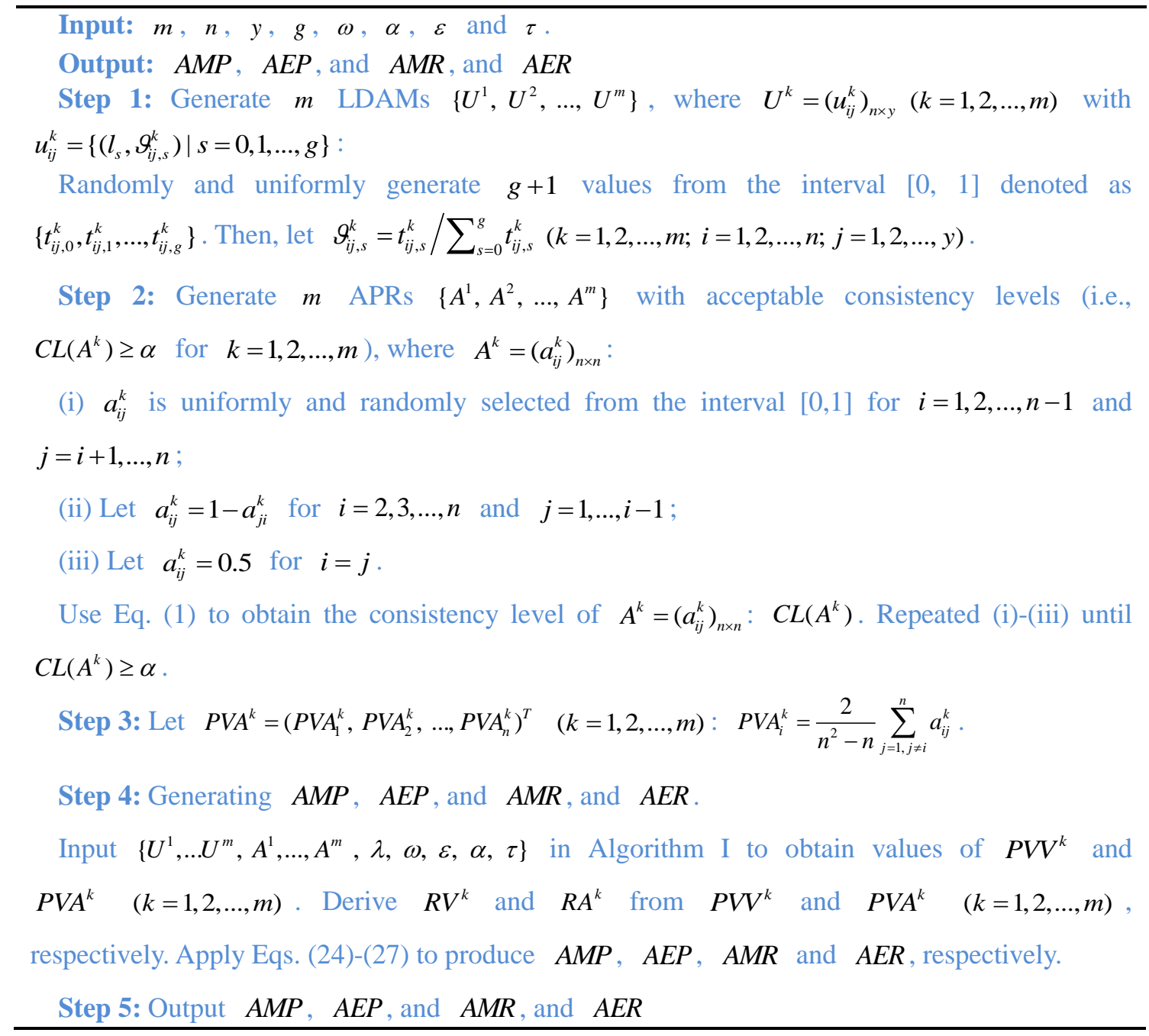

(3) Simulation method II

Simulation method II is obtained by replacing Step 4 of Simulation method I with Step 4' below.

Step 4': Generating $A M P, A E P$, and $A M R$, and $A E R$.

Input $\left\{U^{1}, \ldots U^{m}, A^{1}, \ldots, A^{m}, \lambda, \omega, \alpha, \tau\right\}$ in Algorithm II to obtain the values of $P V V^{k}$ and $P V A^{k}$. Derive $R V^{k}$ and $R A^{k}$ from $P V V^{k}$ and $P V A^{k} \quad(k=1,2, \ldots, m)$, respectively. Apply Eqs. (24)-(27) to produce $A M P, A E P, A M R$ and $A E R$, respectively. 